\title{
Testosterone improves muscle fiber asset and exercise performance in a metabolic syndrome model
}

Erica Sarchielli', Paolo Comeglio², Sandra Filippi3, Ilaria Cellai², Giulia Guarnieri1, Daniele Guasti¹, Elena Rapizzi², Giulia Rastrelli2, Daniele Bani1, Gabriella Vannelli1, Linda Vignozzi2,4,5, Annamaria Morelli1 and Mario Maggi2,4,6

\begin{abstract}
${ }^{1}$ Anatomy and Histology Unit, Department of Experimental and Clinical Medicine, University of Florence, Florence, Italy University of Florence, Florence, Italy

4I.N.B.B. (Istituto Nazionale Biostrutture e Biosistemi), Rome, Italy

${ }^{5}$ Andrology, Women's Endocrinology and Gender Incongruence, Careggi Hospital, Florence, Italy

${ }^{6}$ Endocrinology, Careggi Hospital, Florence, Italy
\end{abstract} 2Sexual Medicine and Andrology Unit, Department of Biomedical, Experimental and Clinical Sciences 'Mario Serio', University of Florence, Florence, Italy ${ }_{3}^{3}$ Interdepartmental Laboratory of Functional and Cellular Pharmacology of Reproduction, Department of Neuroscience, Drug Research and Child Care,

Correspondence should be addressed to A Morelli: a.morelli@unifi.it

\begin{abstract}
Lifestyle modifications, including physical exercise (PhyEx), are well-known treatments for metabolic syndrome (MetS), a cluster of metabolic and cardiovascular risk factors often associated to hypogonadism. Given the trophic role of testosterone on skeletal muscle (SkM), this study was aimed at evaluating the effects of testosterone treatment on SkM metabolism and exercise performance in male rabbits with high-fat diet (HFD)induced MetS. HFD rabbits, treated or not with testosterone (30 mg/ $\mathrm{kg} /$ week) for 12 weeks, were compared to regular diet animals (RD). A subset of each group was exercise-trained for 12 weeks. HFD increased type-II (fast, glycolytic) and decreased type-I (slow, oxidative) muscle fibers compared to RD as evaluated by RT-PCR and histochemistry. Testosterone reverted these effects, also inducing the expression of mitochondrial respiration enzymes and normalizing HFD-induced mitochondrial cristae reduction. Moreover, testosterone significantly increased the expression of myogenic/ differentiation markers and genes related to glucidic/lipid metabolism. At the end of the PhyEx protocol, when compared to RD, HFD rabbits showed a significant reduction of running distance and running time, while testosterone counteracted this effect, also decreasing lactate production. In the trained groups, muscle histology showed a significant reduction of oxidative fibers in HFD compared to RD and the positive effect of testosterone in maintaining oxidative metabolism, as also demonstrated by analyzing mitochondrial ultrastructure, succinate dehydrogenase activity and ATP production. Our results indicate that testosterone could be useful to promote oxidative muscle metabolism altered by MetS, thus improving exercise performance. Conversely, testosterone administration to otherwise eugonadal rabbits (RD) only increased muscle fiber diameter but not endurance performance.
\end{abstract}

\author{
Key Words \\ - testosterone treatment \\ - muscle metabolism \\ - muscle fibers \\ - physical activity \\ - metabolic syndrome
}

Journal of Endocrinology (2020) 245, 259-279 


\section{Introduction}

Metabolic syndrome (MetS) is a clustering of different metabolic alterations and cardiovascular risk factors, with insulin resistance being one of the main pathogenic factors. In men, MetS is also associated with a sex hormone imbalance characterized by low testosterone and gonadotropin levels, leading to hypogonadotropic hypogonadism (HH). The mechanism(s) underlying this association are complex and not fully elucidated. In fact, hormonal imbalance (low testosterone, increased estrogen/testosterone ratio) may be a consequence of metabolic dysregulation, but the converse may also apply with MetS being a consequence of testosterone deficiency (Morelli et al. 2016). Hence, the direction of this association is still undetermined.

The etiology of MetS is multifactorial and comprises both genetic and lifestyle factors (Morelli et al. 2016, Cangiano et al. 2019). The prevalence of MetS is increasing in all age/ethnic groups worldwide, primarily due to modifiable factors such as increased sedentary screen time, physical inactivity and diet (Roberts \& Barnard 2005). Lifestyle modifications, including both dietary and physical training interventions, are well-known treatments for MetS. Exercise and physical activity are recommended for either the primary management of MetS individual risk factors (Pedersen \& Saltin 2015) or for the prevention of MetS itself (Lakka \& Laaksonen 2007). Moreover, physical activity levels and cardiorespiratory fitness display notable inverse relationships with MetS prevalence (Church 2011, Ingle et al. 2017). In particular, a recent review highlighted that aerobic exercise, conducted for a minimum duration of 12 weeks (three times/week), is beneficial for individuals with MetS without diabetes, with improvements in several cardiovascular risk factors (Wewege et al. 2018).

Skeletal muscle is the most abundant tissue in humans and represents $40-60 \%$ of total body weight in normal women and men, with a variety of physiological functions. Muscle loss results, therefore, in both physical alterations and metabolic derangements (Abrigo et al. 2016). Since skeletal muscle accounts for $80 \%$ of insulinstimulated uptake of glucose, it represents one of the principal tissues for evaluating mechanism(s) related to insulin resistance (Stuart et al. 2013).

Muscles have a heterogeneous composition with a mosaic of different fiber types with peculiar physiological contractile properties. Skeletal muscle fibers are primarily classified as 'slow-twitch' (type I) and 'fast-twitch' (type II).
Moreover, based on differential myosin heavy chain (MYH) gene expression, there is a further classification of fast-twitch fibers into three major subtypes (types IIA, IIX, and IIB). Type I fibers primarily use oxidative metabolism and are more resistant to fatigue, while type II fibers partially or completely rely upon glycolytic metabolism ranging from IIA > IIX > IIB (Schiaffino \& Reggiani 2011). Muscle is a versatile tissue and is capable of changing phenotypic properties in response to hormonal, lifestyle and environmental cues. Muscle metabolism and performance depend upon muscle plasticity and, therefore, upon the relative proportion of the fiber types. For example, it was demonstrated that the proportion of slow fibers is significantly increased in response to exercise training (Pette 2002).

Skeletal muscle mass is mainly regulated by exercise training, nutrition and hormonal stimuli that control anabolic-catabolic balance, glucose metabolism, muscle mass maintenance and reparation after injury. It is well known that sex steroids, in particular androgens, promote the growth/maintenance of muscle mass and strength and exert beneficial effects on metabolism and on fiber repair after injury (Carson \& Manolagas 2015). Thus, physiologic or pathologic changes in the levels of circulating sex steroids have important implications for muscle health in both genders (Carson \& Manolagas 2015). Healthy hypogonadal men and individuals with androgen deficiency (as in androgen deprivation therapy for prostate cancer or administration of GnRH agonists) have lower muscle size, strength and function, higher fat mass and higher incidence of insulin resistance, when compared to age-matched eugonadal men (Grinspoon et al. 1996, Bhasin et al. 2004, Gonzalez et al. 2016). Moreover, some studies demonstrated a correlation between the age-associated decline in serum testosterone levels and the reduced mass and strength in male lower limbs (Morley et al. 1997, Baumgartner et al. 1999, Roy et al. 2002). On the contrary, a dose-dependent increase in fat-free mass, muscle size, muscle protein synthesis and maximal voluntary strength was observed both in healthy androgen-deficient (Finkelstein et al. 2013) and in eugonadal men treated with graded doses of testosterone (Bhasin et al. 2004). Accordingly, a recent meta-analysis of randomized controlled trials (RCTs) employing testosterone treatment (TTh) and reporting data on body composition indicates that TTh is able to increase muscle mass and, as a consequence, glucose metabolism (Corona et al. 2016). In particular, TTh determines muscle fiber hypertrophy (i.e. increase in muscle fiber cross-sectional https://joe.bioscientifica.com

https://doi.org/10.1530/JOE-19-0532 (c) 2020 Society for Endocrinology Published by Bioscientifica Ltd.
Printed in Great Britain 
area), but not hyperplasia, with an increase in satellite cell and in myonuclear number (Sinha-Hikim et al. 2002).

In MetS subjects, it has been demonstrated that skeletal muscles had a different arrangement of fibers respect to healthy subjects, with a significant reduction of type I fibers and a resulting muscle composition similar to that of patients with type 2 diabetes (Stuart et al. 2013). In addition, individuals with higher type I fiber content have a reduced risk for developing MetS and diabetes (Hernandez et al. 2001).

Animals fed a high-fat diet (HFD) show an impairment of muscle function and, specifically, the development of the typical features of muscle wasting (such as weakness, loss of muscle mass, decreased fiber diameter) and atrophy (Bhatt et al. 2006, Sishi et al. 2011). In HFD male mice, a decline in type I fibers and an increase in type I/IIA hybrid fibers were observed, suggesting that the type I fibers were transformed primarily into these hybrids and are most susceptible to the effects of obesity (Denies et al. 2014).

During the last 10 years, we set up a nongenetic model of MetS by feeding male rabbits with a high-fat, cholesterol-rich, diet (HFD). The resulting phenotype showed typical features of the human MetS (increased visceral obesity, hypertension, dyslipidemia, glucose intolerance), including hypogonadotropic hypogonadism, with a significant reduction of plasma testosterone and gonadotropins (FSH, LH), along with a decrease in testis and seminal vesicle weights (Filippi et al. 2009). In this experimental model, sex hormone imbalance was related to MetS severity, since testosterone decreased and $\mathrm{E}$ increased as a function of the number of MetS components (Morelli et al. 2016). Interestingly, we recently demonstrated that, when HFD rabbits were trained with aerobic physical activity on a treadmill, they exhibited a reversal of the hypogonadotropic condition, including a restoration of the hypothalamic network controlling GnRH production. However, these animals also showed a deficient muscular response to fatigue, as demonstrated by a significant reduction of both running distance and running time in comparison to the control group fed a regular diet (Morelli et al. 2019). Hence, they are less prone to perform physical exercise. Given the well-known trophic role of testosterone on skeletal muscle (SkM) mass, in this study, we extended the previous findings with the aim of analysing the effects of HFD on SkM composition and metabolism, along with the possible beneficial role of testosterone in preventing these effects and in improving exercise performance in the HFD-induced MetS model.

(C) 2020 Society for Endocrinology Published by Bioscientifica Ltd. Printed in Great Britain

\section{Materials and methods}

\section{Animals' treatment}

Adult male New Zealand White rabbits (Charles River), weighing about $3 \mathrm{~kg}$ (15 weeks old), were individually caged under standard conditions in a temperature and humidity controlled room on a $12 \mathrm{~h}$ light: $12 \mathrm{~h}$ darkness cycle. Water and food were unrestricted throughout the study and food intake was daily measured. After 1 week of regular diet (Harlan-Global Diet 2030, produced by Mucedola s.r.l, Milan, Italy; water $12 \%$, protein $16.5 \%$, fiber $15.5 \%$, ash $8.5 \%$ and vegetable-derived fat $3.5 \%$, total calories $3750 \mathrm{kcal} / \mathrm{kg}$ ), animals were randomly divided into three groups: (1) control rabbits continued to receive a regular diet (RD group; $n=25$ ); (2) treatment rabbits received a high-fat diet (HFD) enriched by $0.5 \%$ animal-derived cholesterol and $4 \%$ peanut oil, total calories $4000 \mathrm{kcal} / \mathrm{kg}$ (HFD group; $n=27$ ), (3) HFD rabbits supplemented with a pharmacological dose of testosterone $(30 \mathrm{mg} / \mathrm{kg}$ weekly i.m.; HFD+testosterone group; $n=25$ ), as previously described (Filippi et al. 2009). To evaluate the effect of testosterone in control animals, an additional group of $\mathrm{RD}$ rabbits was supplemented with a pharmacological dose of testosterone $(30 \mathrm{mg} / \mathrm{kg}$ weekly i.m.; $\mathrm{RD}+$ testosterone group, $n=8$ ) for 12 weeks. A subset of rabbits for each group (RD, $n=10$; HFD, $n=12$; HFD + testosterone, $n=10 ; \mathrm{RD}+$ testosterone $n=4)$ was trained with a physical exercise (PhyEx) running protocol on a treadmill with increasing intensity, duration and frequency during the 12 weeks, as previously published (Morelli et al. 2019) and described below. The remaining animals were left in the cages for the same period. The diet and training began at the same time and lasted 12 weeks. PhyEx rabbits were killed 90 min after the end of the exercise. Blood samples for glucose, total cholesterol, triglycerides, LH and testosterone measurements were collected as previously described (Morelli et al. 2019). In particular, for the PhyEx rabbits, blood samples were obtained immediately before (pre-exercise), immediately after (end exercise) and 90 min after the end (recovery) of the last exercise at week 12 for measuring blood lactate concentration, testosterone, LH, glucose and triglycerides. Mean arterial blood pressure (MAP) was measured and afterward, the rabbits were killed by a lethal dose of pentobarbital (Morelli et al. 2019). After killing, the vastus medialis muscle was rapidly dissected and appropriately stored for the subsequent analyses. To evaluate the prevalence of MetS, we previously designed an algorithm taking into account the presence, as a dummy variable, 
of one or more of the following factors: hyperglycemia, high triglycerides, high cholesterol, increased blood pressure, and visceral fat accumulation. Cutoffs for each factor were derived by the mean \pm two s.D. of the analyzed parameter, as measured in a large database concerning RD rabbits $(n=96)$. Positivity for three or more factors identifies MetS (Maneschi et al. 2012). Animal handling complied with the Institutional Animal Care and Use Committee of the University of Florence, Florence, Italy, which approved the study, in accordance with Italian Ministerial Law, n. 326/2013-B.

\section{Physical exercise protocol}

The physical exercise protocol consisted of a 12-week period of running on a treadmill designed to accommodate rabbits and produced by AL.TO. (Cavezzo, Modena, Italy). During the first 2 weeks, the rabbits ran 2 days/week at a treadmill speed of $10 \mathrm{~m} / \mathrm{min}$ for $3 \mathrm{~min}$ with no inclination $(0 \%)$. From the third week of treatment, the running speed and period were gradually increased with caution: in the third week, the rabbits ran for $5 \mathrm{~min}$ at $10 \mathrm{~m} / \mathrm{min}$ continuously (2 days/week) with inclination (20\%). During subsequent weeks, either the running velocity was extended $5 \mathrm{~m} / \mathrm{min}$ each week until they could run at a speed of $20 \mathrm{~m} / \mathrm{min}$ (from week 6) and the running time was extended 5 min each week until they could run for 25 min per day or until exhausted, with inclination (20\%). From week 4, they were trained for 3 days/week for a total of 9 weeks. Exhaustion was objectively determined when the rabbit refused to run any longer and typically lay down and stretched its hindlimbs. During the exercise, animals were constantly monitored, and when they stopped running, the experiment was immediately considered concluded and the animals were moved to their cages under standard conditions. No adverse events from the exercise were registered.

\section{Plasmatic levels of cholesterol, triglycerides, glucose, testosterone, LH, and lactate}

Plasma cholesterol, triglycerides and glucose levels were measured by using an automated system (ADVIA 2004 Siemens Chemistry System; Siemens Science Medical Solution Diagnostic), as previously described (Filippi et al. 2009). Plasma testosterone and LH levels were measured using a chemiluminescence system (Immunolite 2000 Siemens, Siemens Healthcare Diagnostics) and an ELISA kit (Repropharm Vet, Nouzilly, France), respectively, as previously described (Morelli et al. 2019).
Lactate was measured using an automated system according to the manufacturer's instructions (Lactate Scout, EKF Diagnostic-SensLab, Leipzig, Germany).

\section{Oral glucose tolerance test}

Oral glucose tolerance test (OGTT) was performed as previously published (Filippi et al. 2009). Briefly, after an overnight fasting, a glucose solution $(1.5 \mathrm{~g} / \mathrm{kg})$ was orally administered to the animals and blood samples were collected before and 15, 30, and $120 \mathrm{~min}$ after glucose loading for plasma glucose measurement. The incremental area under the curve (iAUC) was calculated using GraphPad Prism software, version 5.0 for Windows.

\section{RNA extraction and quantitative RT-PCR analysis}

Isolation of total RNA from frozen muscles and RT-PCR analysis were performed as previously described (Morelli et al. 2019). Briefly, for isolation a TRIzol reagent (Life Technologies) and RNeasy Mini Kit (Qiagen) were utilized. cDNA synthesis was carried out using the iScript ${ }^{\mathrm{TM}}$ CDNA Synthesis Kit (Bio-Rad Laboratories). Quantitative realtime RT-PCR (qRT-PCR) amplification and detection were carried out using SsoAdvanced ${ }^{\mathrm{TM}}$ Universal SYBR ${ }^{\circledR}$ Supermix and a CFX96 Two-Color Real-Time PCR Detection System (Bio-Rad Laboratories). Specific PCR primers for rabbit target genes were designed based on sequences available at NCBI GenBank (https://www.ncbi. nlm.nih.gov/genbank/) or Ensemble Genome (http:// www.ensembl.org). The 18S rRNA subunit was used as the reference gene for the relative quantification of the target genes based on the comparative threshold cycle (Ct) $2^{-\Delta \Delta \mathrm{Ct}}$ method.

\section{Histomorphometric analysis, fiber typing and Oil Red $O$ staining}

Some blocks of vastus medialis muscle were embedded in paraffin and used for morphometric evaluation and periodic acid-Schiff (PAS) staining. In detail, deparaffinized and rehydrated $5 \mu \mathrm{m}$ thick cross-sections were processed using standard hematoxylin-eosin staining, and fiber diameter was calculated using Image J 1.51f software (National Institutes of Health) in ten random images from at least three animals for each experimental group. Additional sections were stained with PAS (Bio-Optica S.p.A., Milan, Italy) according to the manufacturer's instructions. Light, medium and dark PAS stained fibers 
were counted in ten random images from at least three animals for each experimental group.

Immediately after collection, other tissue blocks from the muscle belly were affixed to a cork disc with OCT support medium (Sakura Fintek Europe, Zoeterwoude, Netherlands), then frozen by immersion in isopentane, kept at the freezing point in liquid nitrogen, and stored at $-80^{\circ} \mathrm{C}$. Transverse muscle cryostat sections $(8-\mu \mathrm{m}$ thick) were stained with a SDH assay or Oil Red O kit, following the manufacturer's instructions (Bio-Optica S.p.A.). The number of SDH positive fibers was counted in ten random images from at least three animals for each experimental group.

\section{Immunohistochemistry}

Deparaffinized and rehydrated 5- $\mu$ m-thick sections of vastus medialis muscle were processed using immunohistochemical procedures. Briefly, sections were incubated overnight at $4^{\circ} \mathrm{C}$ with mouse monoclonal antiPAX-7 primary antibody (1:50 dilution; sc-81975, Santa Cruz Biotechnology) or anti-MYH3 primary antibody (1:30 dilution; sc-53091, Santa Cruz Biotechnology) and subsequently with biotinylated anti-mouse (Thermo Scientific) followed by streptavidin-peroxidase complex (Thermo Scientific). The reaction product was developed with $3^{\prime}, 3^{\prime}$-diaminobenzidine tetrahydrochloride as chromogen (Sigma-Aldrich). Negative controls were performed avoiding primary antibody. Slides were evaluated and photographed using a Nikon MicrophotFXA microscope. The number of PAX-7 positive cells was counted in ten fields from three animals for each group and expressed as a percentage of the $\mathrm{RD}$ group (mean \pm S.E.M.).

\section{Electron microscopy}

Skeletal muscle specimens were fixed in $4 \%$ glutaraldehyde in $0.1 \mathrm{M}$ cacodylate buffer, $\mathrm{pH} 7.4$, for $24 \mathrm{~h}$, post-fixed in $1 \%$ osmium tetroxide in $0.1 \mathrm{M}$ phosphate buffer, $\mathrm{pH}$ 7.4, for $1 \mathrm{~h}$, dehydrated in graded acetone and embedded in Epon 812 epoxy resin (Fluka, Buchs, Switzerland). Ultrathin sections were stained with uranyl acetate and alkaline bismuth sub nitrate and examined under a JEM 1010 electron microscope (JEOL) at $80 \mathrm{kV}$. Micrographs were taken at $\times 50,000$ final magnification using a Mega View III digital camera (SIS-Soft Imaging System, Munster, Germany) in at least ten fields for each sample. Mitochondrial and internal cristae surface area was measured using iTEM image analysis software (SIS-Soft).

\section{Triglycerides content}

Triglycerides content from skeletal muscle biopsies in six animals from each experimental group was evaluated using the Triglyceride Quantification Colorimetric/Fluorometric Kit (BioVision), following the manufacturer's instructions. Briefly, $100 \mathrm{mg}$ of frozen tissue was mechanically homogenized and resuspended in $1 \mathrm{~mL}$ solution containing 5\% NP-40 in water, heated to $80-100^{\circ} \mathrm{C}$ to solubilize all triglycerides and centrifuged at top speed for $2 \mathrm{~min}$. Lipase was subsequently added and incubated for $20 \mathrm{~min}$ at room temperature to convert triglycerides to glycerol and fatty acids. After the addition of Triglyceride Reaction Mix, absorbance at $570 \mathrm{~nm}$ was read with a plate reader (Victor3 1420, Perkin Elmer).

\section{Tissue homogenates and lysates preparation}

Homogenates were prepared, and succinate dehydrogenase (SDH) activity was measured as previously described (see below and Rapizzi et al. 2015). Small frozen skeletal muscle sections were first chopped finely, and samples were then homogenized in a hand-held plastic homogenizer (80 strokes), in a solution containing $120 \mathrm{mM} \mathrm{KCl}, 20 \mathrm{mM}$ HEPES, $2 \mathrm{mM} \mathrm{\textrm {Mgl } _ { 2 }}, 1 \mathrm{mM}$ EGTA and $5 \mathrm{mg} / \mathrm{mL}$ BSA. Homogenates were centrifuged at $800 \boldsymbol{g}$ for $10 \mathrm{~min}$ at $4^{\circ} \mathrm{C}$, and the enzymatic assay was performed on the supernatant. For lysates, pieces of finely chopped skeletal muscle were lysed in buffer containing $50 \mathrm{mM}$ Tris-HCl pH = 7.5, $120 \mathrm{mM} \mathrm{NaCl}, 1 \mathrm{mM} \mathrm{EGTA}, 6 \mathrm{mM}$ EDTA, $15 \mathrm{mM} \mathrm{Na} \mathrm{P}_{2} \mathrm{O}_{7}, 20 \mathrm{mM} \mathrm{NaF}$ and $1 \%$ Triton X-100 protease inhibitor cocktail. Lysates were clarified by centrifugation at $10,000 \boldsymbol{g}$ for $15 \mathrm{~min}$ at $4^{\circ} \mathrm{C}$. All passages were carried out on ice. All supernatants were quantified for protein content (Coomassie Blue reagent was from Bio-Rad Laboratories).

\section{Western blot analysis}

Lysates $(25 \mu \mathrm{g}$ of protein) were separated by SDS/PAGE and transferred onto PVDF (Immobilon, Millipore), as previously described (Rapizzi et al. 2015). Bound antibodies were detected using ECL reagents (Immobilon, Millipore) and analyzed with a Bio-rad ChemiDoc Imaging System for dedicated chemiluminescent image acquisition (Quantity-One Imaging and Analysis Software by Bio-Rad). Primary antibodies against subunit A of SDH (1:5000 dilution; ab14715, Abcam), actin (1:5000 dilution; sc-1615, Santa Cruz Biotechnology) and TOM20 (1:5000 dilution; cat. 612278, BD Biosciences) were used. 
Secondary antibodies were obtained from Santa Cruz Biotechnology (anti-mouse sc-2005, anti-goat sc-2020).

\section{SDH activity}

Tissue homogenates $\left(\begin{array}{lll}50 & \mu \mathrm{g}\end{array}\right)$ were incubated in a phosphate buffer containing sodium azide, 2,6-dichlorophenolindophenol (DCPIP), sodium succinate and phenazine methosulfate. Complex II specific activity was evaluated by photometry, using the Victor ${ }^{3} 1420$ Multilabel Counter (Packard Instruments, Perkin-Elmer), measuring the decrease in absorbance due to oxidation of DCPIP at $600 \mathrm{~nm}$ (Rapizzi et al. 2015).

\section{Intracellular ATP}

ATP levels were measured using CellTiter-Glo luminescent cell viability assay (Promega). Briefly, muscle homogenates $(25 \mu \mathrm{g} / 50 \mu \mathrm{L})$ were plated in duplicates in a 96-well plate, and an equal volume of CellTiter-Glo reagent was added. Suspensions were shaken at $300 \mathrm{rpm}$ on an orbital shaker for $5 \mathrm{~min}$ and then incubated at room temperature for $25 \mathrm{~min}$ to stabilize the luminescent signal. Luminescence was measured using the Victor $^{3}$ 1420 Multilabel Counter (Packard Instruments, Perkin-Elmer).

\section{Statistical analysis}

Statistical analysis was performed with a one-way ANOVA test (Kruskal-Wallis) followed by Mann-Whitney post hoc analysis or with unpaired two-sided Student's $t$-test, respectively, for non-normally and normally distributed parameters, to evaluate differences between groups. Results are expressed as mean \pm s.E.M. for normally distributed parameters and median with interquartile range for parameters with non-normal distributions. Correlations were assessed using Spearman's methods. Multilevel mixed-effects linear regressions were used for assessing the acute effect of physical exercise over the running and the recovery time in the experimental groups. The models, nested within the animals, were fitted using the treatment group (RD+PhyEx, HFD+PhyEx or HFD + testosterone + PhyEx), time of exercise (pre-exercise, end exercise and recovery) and their interaction term as the independent variables and the serum parameters as the dependent variables. A $P$ value $<0.05$ was considered as significant in all the analyses. Statistical analysis was performed with Statistical Package for the Social Sciences (SPSS v.25.0; SPSS Inc.).
Experimental procedures were carried out using the facilities of the Molecular Medicine Facility, Department of Biomedical Experimental and Clinical Sciences 'Mario Serio', University of Florence.

\section{Results}

\section{MetS phenotype induced by HFD}

HFD rabbits showed a significant increase in all MetS components. In fact, as shown in Table 1, they have hyperglycemia, glucose intolerance, dyslipidemia, hypertension and increase in visceral fat. More than three MetS components were present in $72.7 \%$ of HFDtreated rabbits, whereas it was completely absent in $\mathrm{RD}$ rabbits with or without PhyEx $(P<0.0001)$. Testosterone supplementation to HFD rabbits significantly decreased the percentage of rabbits satisfying MetS criteria (9.1\%, $P=0.002)$. This percentage was further reduced to 0\% in HFD rabbits performing PhyEx and treated with testosterone (Table 1). PhyEx alone numerically reduced the percentage of MetS rabbits (66.7\%), but the difference was not statistically significant from the HFD group $(P=0.79)$.

The occurrence of a HFD-associated hypogonadotropic hypogonadism was demonstrated by the significant reduction of both circulating testosterone and LH levels (Table 1). Treatment with testosterone (TTh; $30 \mathrm{mg} / \mathrm{kg} /$ week for 12 weeks) did not affect HFD-induced dyslipidemia, while significantly ameliorated MAP, and exerted a remarkable positive effect on fasting glucose levels, glucose tolerance, and visceral adipose tissue accumulation (Table 1). As expected, in testosterone-treated rabbits, LH levels remained suppressed (Table 1). Moreover, no significant differences were observed concerning body weight measurements among the different groups (Table 1). Daily food intake in HFD-fed rabbits was not statistically different, as compared to RD-fed rabbits (127.85 g (108.04-150.48) and 138.7 g (121.7.50-155.61), respectively; $P=0.154)$.

\section{Effects of HFD and TTh on skeletal muscle composition}

To characterize the fiber type composition of skeletal muscle samples from each experimental group, we analyzed the mRNA expression of myosin heavy chain (MYH) isoforms, responsible for the contractile properties. We therefore investigated the four fiber types in 

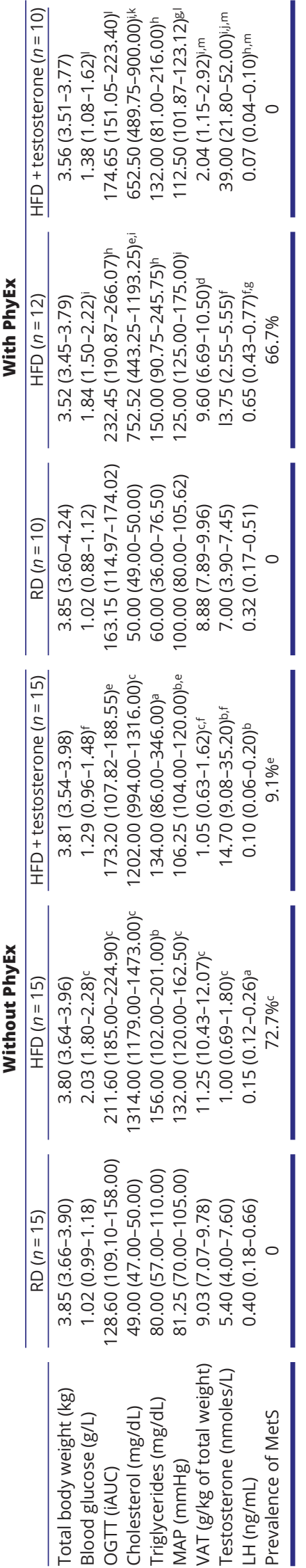

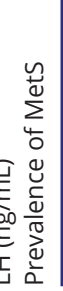

mammalian muscle (type I, IIA, IIX, and IIB), according to the expression of MYH isoform: MYH4 for IIB (very fast/glycolytic), MYH1 for IIX (fast/glycolytic), MYH2 for IIA (fast/oxidative) and MYH7 for type I (slow/oxidative). As shown in Fig. 1, mRNA levels of MYH4 and MYH2 (isoforms specific for type IIB and IIA fibers, respectively) were significantly increased in HFD rabbits when compared to RD group (Fig. $1 \mathrm{~A}$ and $\mathrm{C} ; \mathrm{P}<0.05$ ). In contrast, HFD muscle samples showed a significant decrease of the type I marker MYH7 (Fig. 1D; $P<0.05$ vs RD). TTh significantly counteracted the HFD-induced changes for both MYH4 and MYH7 mRNA expression (Fig. $1 \mathrm{~A}$ and $\mathrm{D} ; P<0.001$ and $P<0.05$ vs HFD, respectively), while increased MYH1 and further increased MYH2 mRNA (Fig. 1B and C; $P<0.05$ and $P<0.001$, respectively). Moreover, the TBX15 gene, encoding a transcription factor specifically expressed in glycolytic myofibers, resulted increased in HFD samples (Fig. 1E; $P<0.05$ vs RD) and decreased up to the control level by TTh (Fig. 1E; $P<0.05$ vs HFD).

Histomorphological analysis by PAS staining of muscle cross-sections confirmed results from mRNA expression. Based on PAS staining intensity, indicative of glycogen content, fibers were classified as light-, mediumor dark-stained, ranging from oxidative to glycolytic. The staining showed a pronounced shift from light PAS-stained (oxidative) to medium/dark PAS-stained (glycolytic) fibers in muscle sections from HFD animals (Fig. 2). In particular, HFD muscles exhibited a significant reduction of light PAS-stained fibers $(P<0.001)$ and a significant increase in medium PAS-stained fibers $(P<0.01)$, as compared to RD muscles (Fig. 2D). TTh significantly increased lightstained fibers (Fig. 2D; $P<0.05$ vs RD, $P<0.001$ vs HFD) and decreased medium-stained fibers (Fig. 2D; $P<0.05$ vs $\mathrm{RD}, P<0.001$ vs HFD). These changes in fiber type composition were confirmed by measuring SDH enzyme activity as marker of oxidative fibers using a specific staining on muscle sections (Fig. 2E, F, G and H). In HFD muscle sections, the percentage of SDH-positive fibers was significantly reduced as compared to RD samples (Fig. $2 \mathrm{H} ; \mathrm{p}<0.001$ ) and significantly increased by TTh (Fig. $2 \mathrm{H} ; \mathrm{P}<0.01$ ).

We next investigated the effect of HFD and TTh on fiber diameter and markers of muscle atrophy and regeneration. As shown in Fig. 3, HFD reduced fiber diameter $(P<0.001$ vs RD; Fig. $3 \mathrm{~A})$ and increased the mRNA expression of the atrophy-related genes, such as FBX032/Atrogin $1 \quad(P<0.01$ vs $\mathrm{RD}$; Fig. $3 \mathrm{~B})$ and MURF1 $(P<0.01$ vs RD; Fig. $3 C)$. TTh counteracted the HFD-related reduction of fiber diameter $(P<0.001$ vs HFD, $\quad P<0.05$ vs RD; Fig. 3A) and normalized 

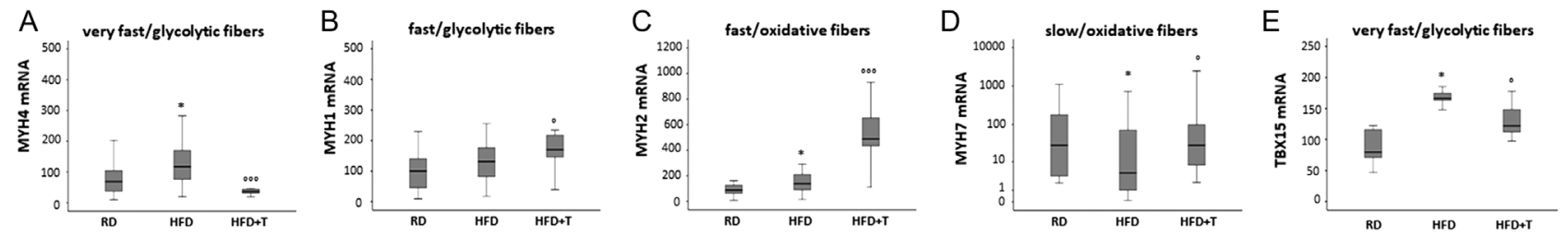

Figure 1

Effect of HFD and TTh on mRNA expression of fiber type markers. (A, B, C and D) mRNA expression of MYH isoforms: MYH4 for type IIB/very fast glycolytic fibers (A), MYH1 for type IIX/fast glycolytic fibers (B), MYH2 for type IIA/fast oxidative fibers (C) and MYH7 for type I/slow oxidative fibers (D). (E) mRNA expression of the fast glycolytic myofiber marker TBX15. The analyses were performed by quantitative RT-PCR in rabbit skeletal muscle from RD, HFD and HFD + testosterone groups. Data were calculated using the $2^{-\triangle \Delta C t}$ comparative method, with the $18 \mathrm{~S}$ ribosomal RNA subunit used as reference gene for normalization, and are reported as mean \pm s.E.M. ( $n=15$ for each group). Statistical analysis between groups was performed with Kruskal-Wallis and post hoc Mann-Whitney non-parametric tests ( ${ }^{*} P<0.05$ vs RD; ${ }^{\circ} P<0.05,{ }^{\circ 0}{ }^{\circ} P<0.001$ vs HFD).

FBX032 mRNA expression up to RD level $(P<0.001$ vs HFD; Fig. 3B). Moreover, TTh significantly reduced MURF1 mRNA expression as compared to HFD $(P<0.05 ;$ Fig. 3C). Interestingly, MYH4 and TBX15 expression (markers of glycolitic fibers) was positively associated with expression of FBX032/Atrogin 1 mRNA $(r=0.408, P=0.015, n=35$ and $r=0.458, P=0.049, n=19$, respectively).

On the other side, the myogenesis-related gene myogenin (MYOG) was significantly decreased in HFD muscles (Fig. 3D; $P<0.001$ vs RD) and restored by TTh (Fig. 3D, $P<0.01$ vs HFD). TTh also upregulated the mRNA expression of two other myogenesis markers, such as MYF5 (Fig. 3E, $P<0.001$ vs RD and HFD) and PAX7 (Fig. 3F, $P<0.01$ vs RD and HFD) and the muscle differentiation markers MYOD (Fig. $3 \mathrm{~K}, P<0.01$ vs RD and HFD) and ITGA7/ $\alpha 7$ integrin (Fig. $3 \mathrm{~L}, P<0.001$ vs RD and HFD). The myogenic effect of TTh was confirmed by immunohistochemistry of PAX7 expression in skeletal muscle sections from each rabbit group. As shown in Fig. 3 (panels G, H, I and J), we found a significant increase of PAX7-positive cells in muscle sections from testosteronetreated rabbits, as compared to both RD and HFD groups $(P<0.001)$. Moreover, using an antibody against MYH3, which is expressed by newly generated myofibers, we detected MYH3-positive fibers exclusively in muscle sections from testosterone-treated HFD rabbits (Fig. 3M).

Accordingly, a positive association was found between circulating testosterone and skeletal muscle expression of several genes related to muscle regeneration (MYOG, MYF5, PAX7, ITGA7), mitochondrial and oxidative metabolism (SLC25A12, NDUFB5, UCP2), while the expression of the

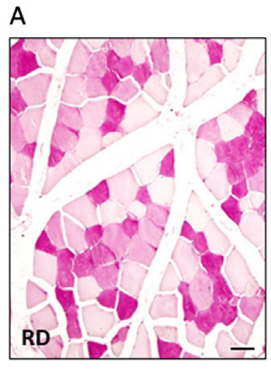

E

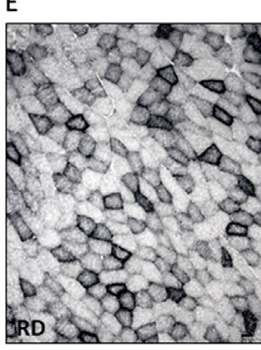

B
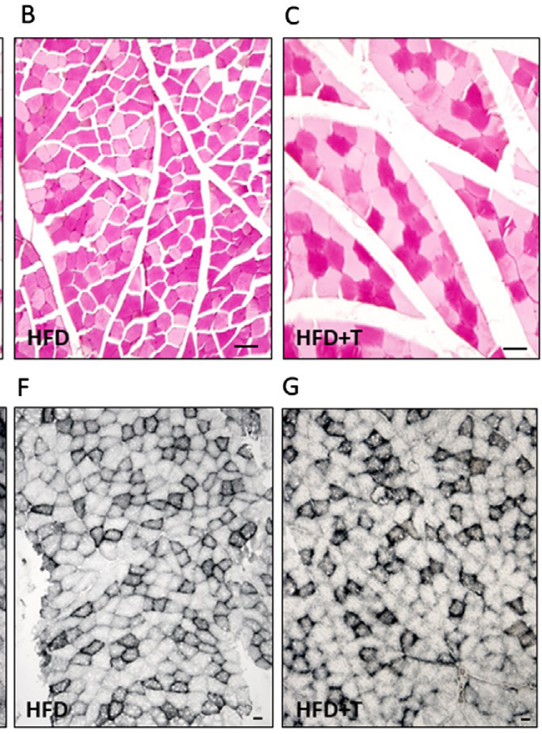

G

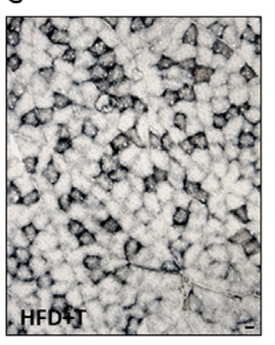

D

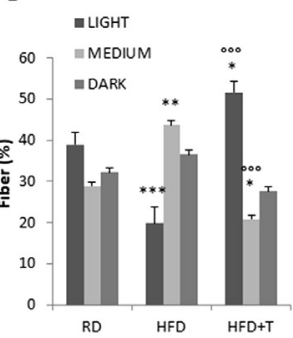

$\mathrm{H}$

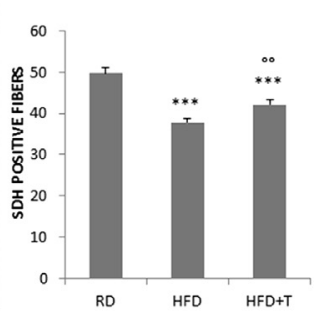

Figure 2

Effect of HFD and TTh on muscle fiber composition. (A, B and C) Representative images of PAS-stained vastus medialis cross-sections from RD (A), HFD (B) and HFD + testosterone (C) rabbits showing a shift toward glycolytic more intense stained fibers in HFD samples compared to RD, that was reverted by TTh; (D) quantification of light, medium and dark stained fibers calculated by counting ten fields from muscle sections of at least three animals for each experimental group. (E, F and G) Representative images of SDH enzymatic assay in vastus medialis cryo-sections from RD (E), HFD (F) and $\mathrm{HFD}+$ testosterone $(\mathrm{G})$ rabbits showing a reduction of SDH activity in HFD sections, significantly counteracted by TTh. $(\mathrm{H})$ Quantification of SDH-positive fibers calculated by counting ten fields of at least three animals for each experimental group. Statistical analysis between groups was performed with unpaired two-sided Student's $t$-test $(* P<0.05, * \star P<0.01$, $* * * P<0.001$ vs RD; ${ }^{\circ} P<0.01,{ }^{\circ 00} P<0.001$ vs HFD). Scale bar $50 \mu \mathrm{m}$. A full colour version of this figure is available at https://doi.org/10.1530/ JOE-19-0532. https://joe.bioscientifica.com https://doi.org/10.1530/JOE-19-0532
(C) 2020 Society for Endocrinology Published by Bioscientifica Ltd.
Printed in Great Britain 
B
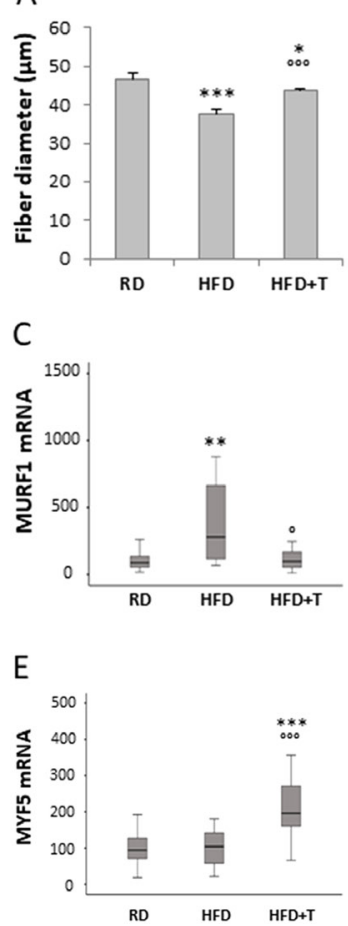

D

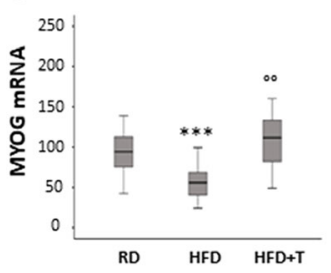

F
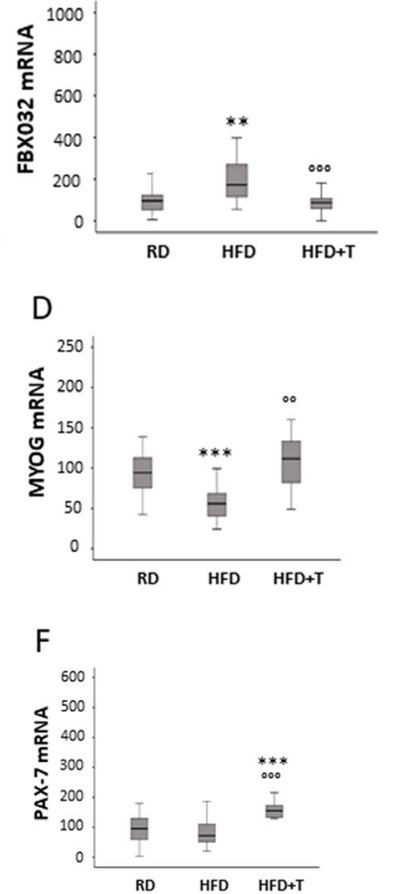

G
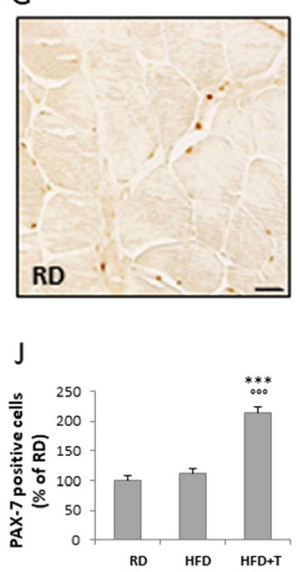

$\mathrm{M}$

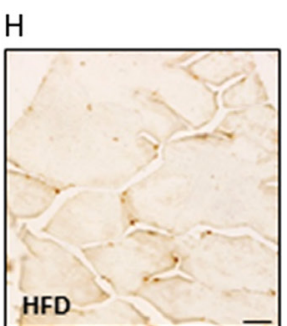

I

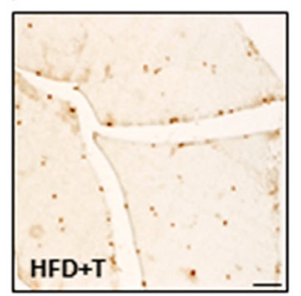

L

K L
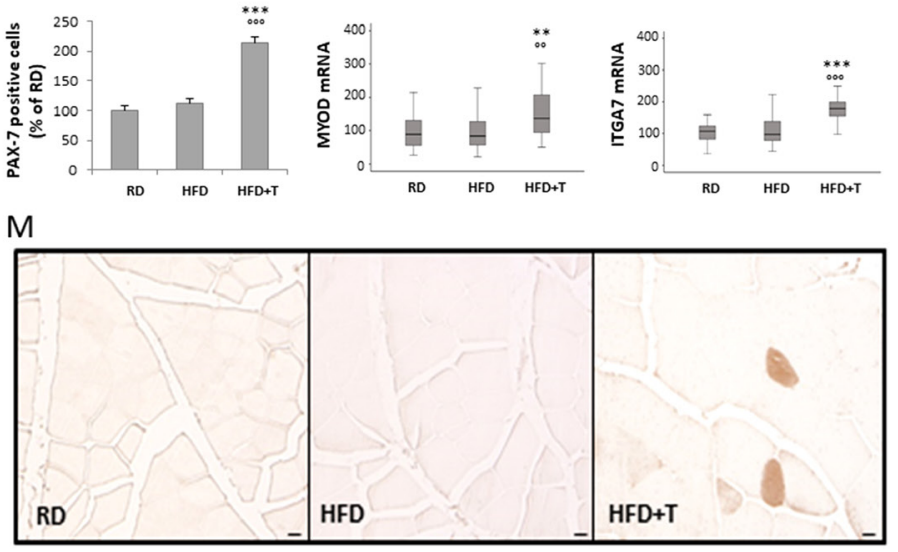

Figure 3

Effect of HFD and TTh on fiber size and muscle regeneration/differentiation. (A) Fiber diameter, as measured in muscle cross-sections of specimens from RD, HFD and HFD + testosterone rabbits, was reduced by HFD, while TTh counteracted this effect. Data were calculated by Image $1.51 \mathrm{f}$ software in ten fields from at least three animals for each experimental group and expressed as mean \pm S.E.M. Statistical analysis between groups was performed with unpaired two-sided Student's $t$-test ( $* P<0.05$ and $* * * P<0.001$ vs RD, ${ }^{\circ 0} p<0.001$ vs HFD). (B, C, D, E, F and G) TTh normalized the HFD-altered mRNA expression of atrophy-related genes, such as FBX032/Atrogin-1 (B) and MURF-1 (C), and the myogenesis-related gene MYOG (D). Also the muscle regeneration markers MYF5 (E) and PAX7 (F), the muscle differentiation markers MYOD (K) and ITGA7/ $\alpha 7$ integrin (L) were significantly increased by TTh. Data were calculated using the 2- $\Delta \Delta \mathrm{ct}$ comparative method, with the $18 \mathrm{~S}$ ribosomal RNA subunit used as reference gene for normalization and are reported as mean \pm S.E.M. ( $n=15$ for each group). Statistical analysis was performed with Kruskal-Wallis and post hoc Mann-Whitney non-parametric tests $\left(* P<0.05, * * P<0.01, * * * P<0.001\right.$ vs RD; ${ }^{\circ} P<0.01,{ }^{\circ 0 \circ} P<0.001$ vs HFD). (G, $\mathrm{H}, \mathrm{I}$ and $\mathrm{J}$ ) Immunohistochemical analysis of PAX-7 in vastus medialis muscle cross-sections from RD (G), HFD (H) and HFD + testosterone (I) rabbits; (J) quantification of PAX-7-positive cells counted in ten fields from three animals for each group and expressed as percentage of RD (mean \pm S.E.M.), showing a significant increase of PAX-7 expression in sections from testosteronetreated HFD rabbits (scale bar $20 \mu \mathrm{m}$ ). Statistical analysis between groups was performed with unpaired two-sided Student's $t$-test ( $* \star \star P<0.001$ vs RD; ${ }^{\circ \circ} \mathrm{P}<0.001$ vs HFD). (M) Representative images of the muscle regeneration marker MYH3 in vastus medialis cross-sections, as evaluated by immunohistochemical analysis: MYH3-positive fibers are detected exclusively in HFD + testosterone sample (scale bar $20 \mu \mathrm{m}$ ). A full colour version of this figure is available at https://doi.org/10.1530/JOE-19-0532.

type IIB marker MYH4 and atrophy marker FBX032 was negatively associated with testosterone levels (Table 2).

As reported in Fig. 4, TTh effect on oxidative function was confirmed by the significant induction of the expression of some mitochondrial respiration-related genes, such as NADH dehydrogenase ubiquinone 1 beta subcomplex 3 and 5 (NDUFB3 and NDUFB5; $P<0.001$ and $P<0.01$ vs both $\mathrm{RD}$ and HFD, respectively; Fig. $4 \mathrm{~A}$ and $\mathrm{B}), \mathrm{NADH}$-ubiquinone oxidoreductase subunit 1 (NDUFS1; $P<0.05$ vs both RD and HFD; Fig. 4C), succinate dehydrogenase (SDHB; $P<0.01$ vs both RD and HFD; Fig. 4D) and citrate synthase (CS; $P<0.01$ vs, RD, $P<0.05$ vs HFD; Fig. 4E). Accordingly, transmission electron microscopy studies evidenced mitochondrial dysfunctions in HFD muscle samples, with pronounced reduction of the internal cristae surface area, as compared to RD (Fig. 4F, G and I; $P<0.01$ vs RD). TTh counteracted the HFD-induced modifications of the mitochondrial cristae (Fig. $4 \mathrm{H}$ and I; $P<0.05$ vs HFD).

To further characterize skeletal muscle metabolism, we analyzed the mRNA expression of genes related to insulin sensitivity, lipid and energy metabolism (Fig. 5). We found that TTh induced a significant increase in mRNA expression of insulin receptor substrate-1 (IRS-1; $P<0.001$ vs $\mathrm{RD}$ and HFD; Fig. 5A), lipoprotein lipase (LPL; $P<0.01$ vs RD, $P<0.05$ vs HFD; Fig. 5B), peroxisome proliferator-activated receptor- $\alpha(\operatorname{PPAR} \alpha ; P<0.001$ vs RD and HFD; Fig. 5C), uncoupled protein-2 (UCP2; $P<0.001$ vs RD and HFD; Fig. 5D) and UCP3 ( $P<0.01$ vs RD, $P<0.05$ vs HFD; Fig. 5E). Interestingly, the brown adipose 
Table 2 Association between gene expression in skeletal muscle samples and circulating testosterone.

\section{Gene}

MYF5 (myogenic factor 5)

PAX7 (paired box 7)

MYOG (myogenin)

ITGA7 (skeletal muscle transcripts of the human integrin alpha7)

SLC25A12 (solute carrier family 25 aspartate/glutamate carrier, member 12)

NDUFB5 (NADH dehydrogenase ubiquinone 1 beta subcomplex 5)

MYH4 (myosin, heavy chain 4)

FBX032 (muscle atrophy F-box)

UCP2 (uncoupling protein 2)

$\begin{array}{r}\hline \boldsymbol{r} \\ \hline 0.537 \\ 0.390 \\ 0.472 \\ 0.539 \\ 0.386 \\ 0.444 \\ -0.424 \\ -0.368 \\ 0.516 \\ \hline\end{array}$

\begin{tabular}{c}
$P$ \\
\hline 0.000 \\
0.008 \\
0.003 \\
0.000 \\
0.009 \\
0.002 \\
0.004 \\
0.013 \\
0.000
\end{tabular}

\begin{tabular}{c}
\hline $\boldsymbol{n}$ \\
\hline 45 \\
45 \\
38 \\
45 \\
45 \\
45 \\
45 \\
45 \\
45
\end{tabular}

Results derived from univariate correlations and are presented as Spearman coefficients. Bold indicates statistical significance.

tissue-specific UCP1 mRNA was significantly reduced by HFD $(P<0.05$ vs RD; Fig. $5 \mathrm{~F})$ and normalized by TTh $(P<0.01$ vs HFD; Fig. 5F). Moreover, Oil Red O staining showed that skeletal muscle cryosections from HFD rabbits presented a lipid intramyocellular accumulation (Fig. 5H), which was not present in muscle samples from RD, where a light lipid positivity was localized to intermuscular spaces (Fig. 5G). These results were paralleled by a significant increase of triglyceride content in HFD muscle extracts as compared to RD group ( $P<0.05$; Fig. 5J). Interestingly, TTh normalized both lipid content localization (Fig. 5I) and triglyceride content $(P<0.05$ vs HFD; Fig. 5J).

\section{Effect of physical exercise on skeletal muscle composition and metabolism}

Since we observed alterations in skeletal muscle induced by HFD and ameliorated by TTh, we next evaluated the
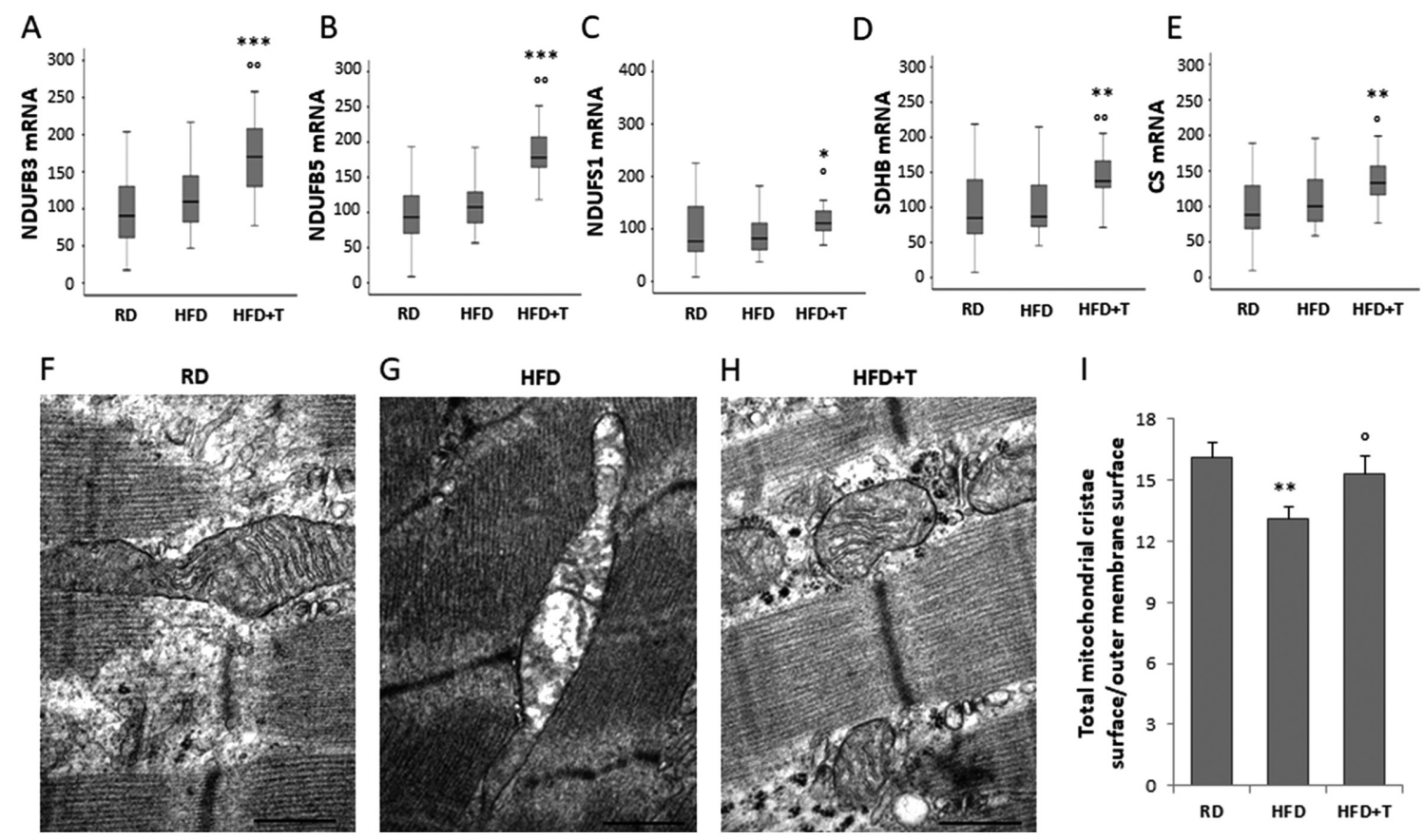

\section{Figure 4}

Effect of HFD and TTh on mitochondrial respiration-related gene expression and mitochondrial cristae aspect. (A, B, C, D and E) Quantitative RT-PCR analysis shows a positive effect of TTh on mRNA expression of the mitochondrial respiration-related genes NDUFB3 (A), NDUFB5 (B), NDUFS1 (C), SDHB (D) and CS (E). Data were calculated using the 2- $\triangle \Delta \mathrm{Ct}$ comparative method, with the $18 \mathrm{~S}$ ribosomal RNA subunit used as reference gene for normalization, and are reported as mean \pm S.E.M. ( $n=15$ for each group). Statistical analysis was performed with Kruskal-Wallis and post hoc Mann-Whitney nonparametric tests ( $* * P<0.01, * \star * P<0.001$ vs RD, ${ }^{\circ} P<0.05,{ }^{\circ} P<0.01,{ }^{\circ}{ }^{\circ} P<0.001$ vs. HFD). (F, G, H and I) Mitochondrial ultrastructure, as visualized by transmission electron microscopy in skeletal muscle specimens, was affected by HFD (G) and normalized by TTh (H). Bar graph (I) represents the ratio between the cristae membrane surface area and the outer membrane surface area as measured using iTEM image analysis software (SIS) in ten fields of muscle sections from at least three animals for each experimental group. Data are reported as mean \pm S.E.M. Statistical analysis was performed with unpaired two-sided Student's $t$-test ( ${ }^{*} * P<0.01$ vs RD; ${ }^{\circ} P<0.05$ vs HFD). Scale bar $500 \mathrm{~nm}$. 

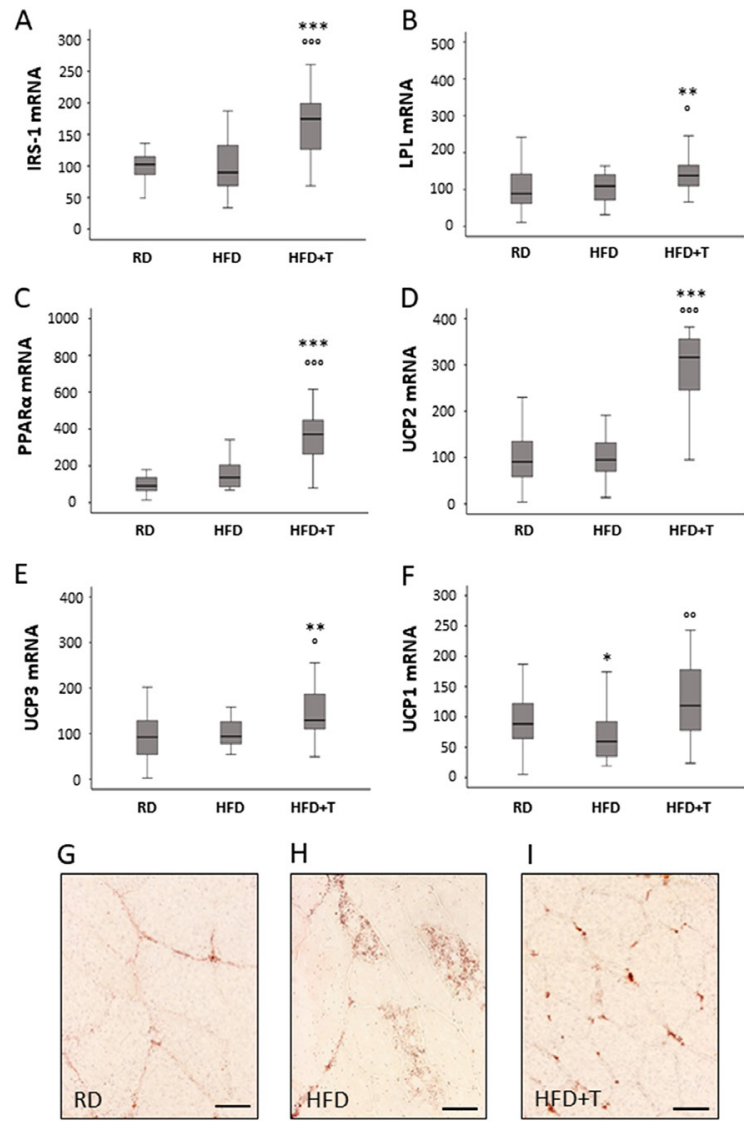

$\mathrm{H}$
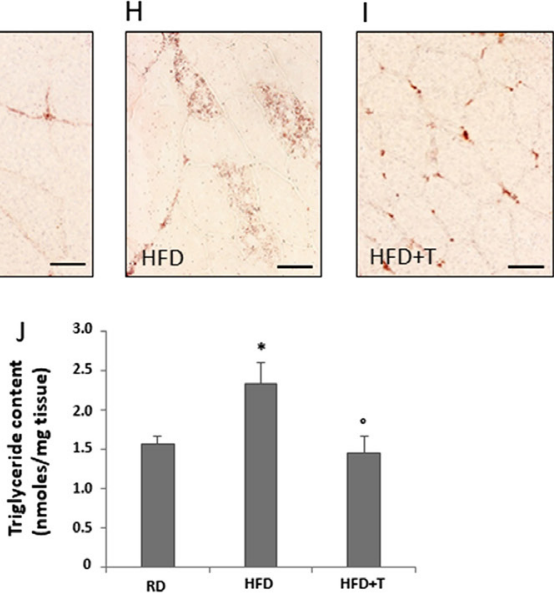

\section{Figure 5}

TTh effect on genes related to insulin sensitivity, lipid and energy metabolism and on lipid accumulation in muscle samples. (A, B, C, D, E and F) TTh induced mRNA expression of IRS-1, gene related to insulindependent signaling (A), as well as LPL (B) and PPAR $\alpha(C)$, genes implicated in the lipid metabolism. TTh also increased the expression of genes involved in energy dissipation as UCP2 (D) and UCP3 (E) and normalized the HFD-altered expression of the brown adipose tissuespecific UCP1 (F). Data were calculated using the 2- $\triangle \Delta C t$ comparative method, with the $18 \mathrm{~S}$ ribosomal RNA subunit used as reference gene for normalization, and are reported as mean \pm S.E.M. ( $n=15$ for each group). Statistical analysis between groups was performed with Kruskal-Wallis and post hoc Mann-Whitney non-parametric tests ( $* P<0.05, * * P<0.01$, $* \star * P<0.001$ vs RD; ${ }^{\circ} P<0.05,{ }^{\circ} P<0.01,{ }^{\circ 00} P<0.001$ vs HFD). (G, H and I) Oil Red $O$ staining shows intramyocellular lipid accumulation in HFD muscle cryosections $(\mathrm{H})$ and intermuscular lipid localization in RD (G) and $\mathrm{HFD}+$ testosterone (I) muscle sections (scale bar $50 \mu \mathrm{m}$ ). (H) TTh normalized the triglycerides content increased by HFD, as evaluated by Triglyceride Quantification Kit on muscle lysates from six animals for each experimental group; data are reported as mean \pm S.E.M. and statistical analysis was performed with unpaired two-sided Student's $t$-test ( ${ }^{*} P<0.05$ vs $\mathrm{RD},{ }^{\circ} P<0.05$ vs HFD). A full colour version of this figure is available at https://doi.org/10.1530/JOE-19-0532.

(C) 2020 Society for Endocrinology Published by Bioscientifica Ltd.
Printed in Great Britain physical endurance and the skeletal muscle functional response in terms of fiber composition and metabolism in all the experimental groups. We trained a subgroup of $\mathrm{RD}, \mathrm{HFD}$ and HFD + testosterone rabbits with a progressive treadmill running protocol for 12 weeks. In HFD rabbits, physical activity significantly improved MetS parameters, such as hypercholesterolemia, percentage of visceral fat and testosterone and LH levels when compared to the untrained rabbits (Table 1). Conversely, no effect was observed in glucose metabolism, triglycerides and mean arterial pressure (Table 1). As expected, in testosterone-treated rabbits, physical activity did not change LH levels that remained suppressed (Table 1). Although the HFD+testosterone+PhyEx group showed numerical improvements in some MetS components (such as blood glucose, MAP and VAT), the differences were not statistically significant, as compared to the HFD + testosterone group (Table 1).

Maximum running time and distance on the treadmill were utilized to assess the endurance capacity of rabbits, exercised on a $20 \%$ slope until unable to run for $10 \mathrm{~s}$. As showed in Table 3, HFD rabbits were able to run for a lower distance and for a shorter time respect to RD rabbits, although the lactate production was similar in the two groups. When lactate production was normalized for running distance, it resulted significantly increased by HFD (Table 3). TTh significantly improved physical resistance to exercise by increasing both maximal running time and distance and normalizing lactate production over running distance (Table 3). Table 3 also shows glycemia levels before and after exercise. In RD+PhyEx at the end of exercise glycemia tends to increase, whereas in HFD + PhyEx rabbits such an increase was not observed, although the pre-exercise levels were higher than in control. TTh partially reduced basal glycemia and allowed a sustained increase in glycemia upon PhyEx.

The effect of endurance training on fiber muscle composition was evaluated by PAS staining and SDH activity assay (Fig. 6). In HFD + PhyEx muscles, PAS staining showed a shift to glycolytic fibers with an increase in both medium and dark staining and a parallel reduction of light stained fibers (Fig. 6A and B) when compared to RD+PhyEx $(P<0.001$ and $P<0.05$ vs $\mathrm{RD}+$ PhyEx, respectively, see bar graph in Fig. $6 \mathrm{D})$. TTh was able to restore all these alterations up to RD+PhyEx levels (Fig. 6C and D). These data were confirmed by measuring SDH enzyme activity with a specific staining on muscle sections (Fig. 6E, F, G and $\mathrm{H}$ ) and with an enzymatic assay from total muscle extracts (Fig. 6I). In HFD+PhyEx samples, 
Table 3 Effects of acute exercise in trained RD, HFD and HFD + testosterone rabbits.

\begin{tabular}{|c|c|c|c|c|c|}
\hline & Pre-exercise & End & $\begin{array}{l}\text { P end vs pre-exercise } \\
\text { within group }\end{array}$ & Recovery & $\begin{array}{l}P \text { recovery vs } \\
\text { pre-exercise } \\
\text { within group }\end{array}$ \\
\hline \multicolumn{6}{|l|}{ Running distance (m) } \\
\hline $\mathrm{RD}$ & --- & $200.00(159.87 ; 308.13)$ & --- & --- & --- \\
\hline HFD & --- & $105.00(76.88 ; 154.78)^{b}$ & --- & --- & --- \\
\hline HFD + testosterone & --- & $225.00(164.87 ; 338.50)$ & --- & --- & --- \\
\hline \multicolumn{6}{|l|}{ Running time (s) } \\
\hline $\mathrm{RD}$ & --- & $600.00(479.62 ; 924.38)$ & --- & --- & --- \\
\hline HFD & --- & $315.00(230.65 ; 464.35)^{\mathrm{b}}$ & --- & --- & --- \\
\hline HFD + testosterone & --- & $675.00(494.60 ; 1015.51)$ & --- & --- & --- \\
\hline \multicolumn{6}{|l|}{ Lactate (nmol/L) } \\
\hline $\mathrm{RD}$ & $3.40(2.38 ; 6.75)$ & $14.55(10.37 ; 19.87)$ & $<0.0001$ & --- & --- \\
\hline HFD & $3.10(1.86 ; 7.83)$ & $18.30(12.42 ; 20.14)$ & $<0.0001$ & --- & \\
\hline HFD + testosterone & $7.20(5.02 ; 10.48)$ & $18.35(14.07 ; 21.73)$ & $<0.0001$ & --- & \\
\hline \multicolumn{6}{|c|}{ Lactate/running distance (nmol/L for each 100 m) } \\
\hline $\mathrm{RD}$ & -- & $6.16(4.15 ; 11.95)$ & --- & --- & --- \\
\hline HFD & --- & $16.18(11.65 ; 22.73)^{b}$ & --- & --- & --- \\
\hline HFD + testosterone & --- & $7.36(5.29 ; 12.11)$ & --- & --- & --- \\
\hline \multicolumn{6}{|l|}{ Blood glucose (g/L) } \\
\hline $\mathrm{RD}$ & $0.95(0.76 ; 1.11)$ & $1.16(0.92 ; 1.48)$ & 0.086 & $1.02(0.91 ; 1.15)$ & 0.553 \\
\hline HFD & $1.60(1.33 ; 2.26)^{c}$ & $1.94(1.65 ; 2.15)^{b}$ & 0.449 & $1.84(1.52 ; 2.29)^{c}$ & 0.537 \\
\hline HFD + testosterone & $1.26(1.08 ; 1.68)$ & $1.73(1.21 ; 2.69)^{\mathrm{b}}$ & $<0.0001$ & $1.38(1.06 ; 1.60)^{a}$ & 0.223 \\
\hline \multicolumn{6}{|l|}{ Triglycerides (mg/dL) } \\
\hline $\mathrm{RD}$ & $59.50(51.50 ; 81.90)$ & $66.00(52.77 ; 86.63)$ & 0.727 & $60.00(43.50 ; 72.50)$ & 0.311 \\
\hline HFD & $139.50(71.04 ; 342.96)^{a}$ & $155.50(67.52 ; 355.65)^{\mathrm{a}}$ & 0.559 & $162.00(72.29 ; 350.38)^{\mathrm{b}}$ & 0.581 \\
\hline HFD + testosterone & $111.50(70.22 ; 217.18)$ & $120.00(62.23 ; 215.77)$ & 0.584 & $132.00(87.89 ; 212.51)$ & 0.449 \\
\hline
\end{tabular}

Measurements were performed before starting (pre-exercise), at the end of the exercise (end) and after 90' from the exercise (recovery). Results derive from multilevel mixed-effect linear regressions (nested within the animals) using the serum parameters as dependent variables and treatment, time of exercise and their interaction term as independent variables. The interaction time $\times$ treatment is reported using RD as referent. Data are reported as median with 95\% confidence intervals (in brackets). RD, $n=10 ; \mathrm{HFD}, n=12$ and HFD + testosterone, $n=10$.

a $P<0.05, \mathrm{~b} P<0.01, c P<0.0001$ vs RD.

the activity of SDH was reduced and restored by TTh (Fig. $6 \mathrm{E}, \mathrm{F}, \mathrm{G}, \mathrm{H}$ and I). Moreover, also the protein expression of the subunit A of SDH, evaluated by Western blotting of vastus medialis lysates, was significantly increased by TTh (Fig. 6J, $P<0.01$ vs either RD + PhyEx or HFD + PhyEx). Similar results were obtained analyzing the protein expression of TOM20, a marker of mitochondrial mass (Fig. 6J). To further demonstrate the positive effect of TTh on mitochondrial respiration, we measured ATP levels in muscle extracts from each experimental trained group. Figure $6 \mathrm{~K}$ shows a significant reduction of ATP levels in HFD + PhyEx samples, as compared to RD + PhyEx $(P<0.01)$, that was completely reverted by TTh $(P<0.01$ vs HFD + PhyEx).

Transmission electron microscopy analysis of mitochondria further demonstrated the negative effects of HFD on cristae that were maintained also in exercise trained rabbits (Fig. 7A and $\mathrm{B} ; P<0.001$ vs $\mathrm{RD}+$ PhyEx); once again TTh was able to ameliorate mitochondrial cristae aspect (Fig. 7C; $P<0.05$ vs HFD + PhyEx).

\section{Effect of testosterone supplementation on skeletal muscle composition and physical endurance in RD group}

To evaluate whether testosterone effects could be detected also in RD animals, independently of the HFD-induced features (including hypogonadism), we analyzed an additional group of eugonadal rabbits fed a RD and treated with testosterone $(30 \mathrm{mg} / \mathrm{kg}$ ) for 12 weeks. As evaluated by both mRNA expression analysis of fiber type markers (Table 4) and PAS staining of muscle cross-sections (Fig. $8 \mathrm{~A})$, we found that testosterone supplementation to RD did not change skeletal muscle fiber types proportion when compared to RD untreated group. No differences were also observed in the expression of mitochondrial oxidative function-related genes (Table 4). In contrast, fiber diameter (Fig. 8B) and mRNA expression of markers of both myogenesis and muscle differentiation, such as PAX7 (Fig. 8D) and ITGA7 (Fig. 8E), respectively, were significantly increased by testosterone supplementation, https://joe.bioscientifica.com https://doi.org/10.1530/JOE-19-0532
(C) 2020 Society for Endocrinology Published by Bioscientifica Ltd. Printed in Great Britain 


\section{Journal of}

A

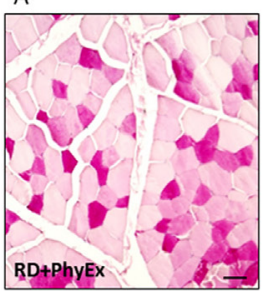

E

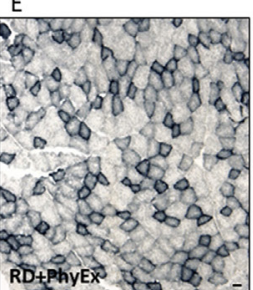

B
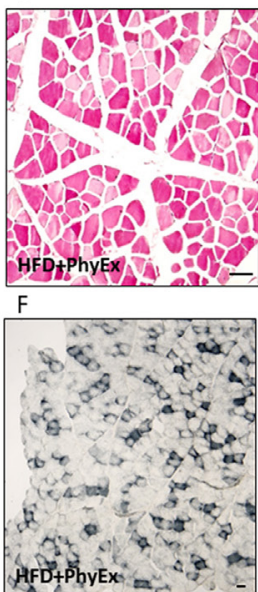
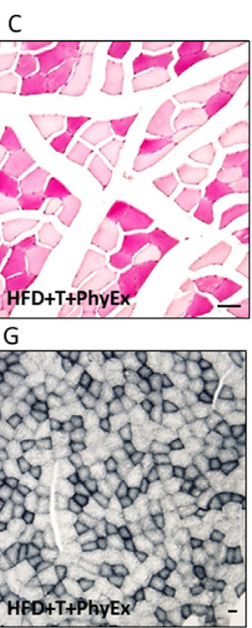

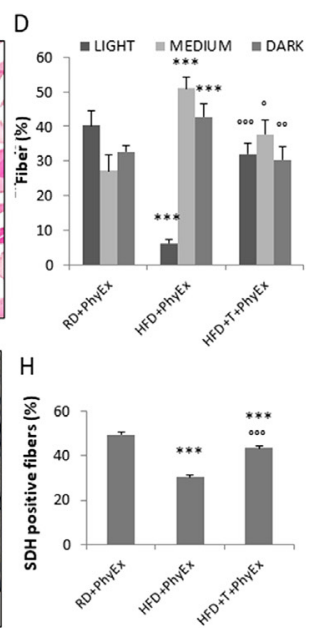

I

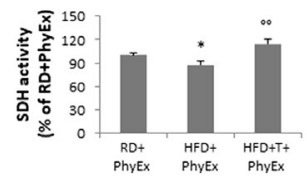

$\mathrm{J}$
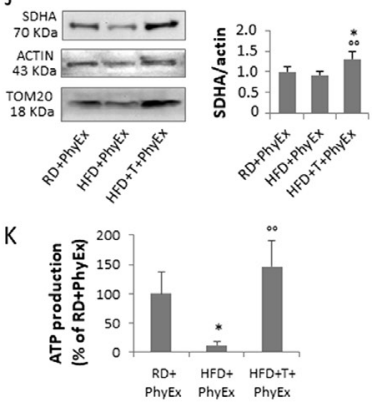

\section{Figure 6}

Effect of PhyEx and TTh on muscle fiber composition in each experimental group. (A, B and C) Representative images of PAS-stained vastus medialis cross-sections from RD (A), HFD (B) and HFD + testosterone (C) treadmill trained rabbits showing a reduction of light stained fibers and a further shift towards medium/dark stained (glycolytic) fibers induced by HFD and normalized by TTh. (D) Quantification of light, medium and dark stained fibers calculated by counting at least ten fields from muscle sections of at least three different animals for each experimental group. (E, F, G, $\mathrm{H}$ and I) SDH enzymatic assay in either vastus medialis cryo-sections or in total muscle extracts shows a reduction of SDH activity in HFD samples, significantly counteracted by TTh. (E, F and G) Representative images of SDH staining in muscle sections. (H) The bar graphs show the quantification of SDH-positive fibers expressed as percentage of total fibers counted in ten fields from at least three different animals for each group. (I) SDH activity in muscle extracts from three animals for each group expressed as percentage of RD + PhyEx, taken as 100\%. (J) Representative immunoblots of SDHA and TOM20 in vastus medialis protein lysates from each experimental group and quantification of SDHA band intensity normalized over actin signal; data are expressed as mean \pm S.E.M. and derive from three different animals for each group. (K) Analysis of ATP levels in muscle lysates from RD, HFD and HFD + testosterone rabbits after PhyEx protocol expressed as percentage over RD + PhyEx, taken as 100\%. Data are from at least six animals for each group. Statistical analysis between groups was performed with unpaired two-sided Student's $t$-test $\left(* P<0.05, * \star \star * P<0.001\right.$ vs RD; ${ }^{\circ} P<0.05,{ }^{\circ} P<0.01,{ }^{\circ 0} P<0.001$ vs HFD). Scale bar $50 \mu \mathrm{m}$. A full colour version of this figure is available at https://doi.org/10.1530/JOE-19-0532.

as compared to RD group $(P<0.001$ vs RD for fiber diameter; $P<0.01$ vs RD for PAX7 and ITGA7 mRNA). Accordingly, circulating testosterone levels were positively associated with fiber diameter (Fig. 8C) and ITGA7 mRNA expression (Fig. 8F). Genes related to glucose (PPAR $\alpha$ and IRS-1) and energy (UCP1, UCP2 and UCP3) metabolism were not affected by testosterone, except for the triglyceride hydrolysis-related gene LPL, that resulted significantly increased $(P<0.001$ vs $\mathrm{RD}$; Table 4$)$. Moreover, physical endurance evaluation of the subset of $\mathrm{RD}+$ testosterone rabbits trained with the treadmill protocol was similar to that of RD group in terms of running distance, running time, lactate production pre- and post-exercise, and lactate/running distance ratio, as reported in Table 5. By analyzing PAS-stained muscle cross-sections, SDH-positive fibers and ATP production in skeletal muscle samples from
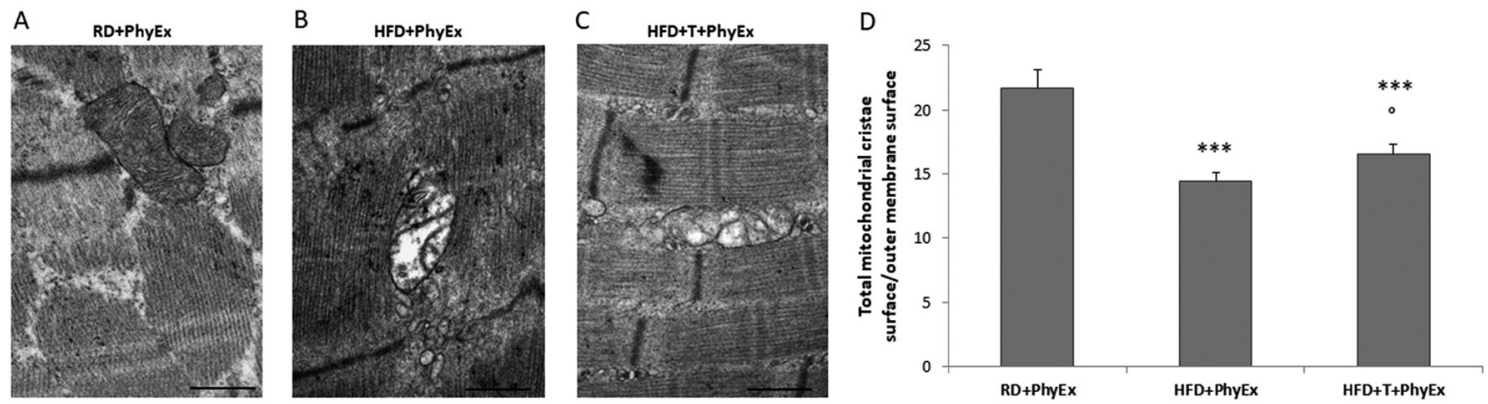

\section{Figure 7}

Effect of PhyEx and TTh on mitochondrial ultrastructure. (A, B and C) Ultrastructural analysis of skeletal muscle specimens showed mitochondrial alterations in HFD samples which were significantly reduced in the samples from TTh-treated animals. (D) Bar graph indicates the ratio between the cristae membrane surface area and the outer membrane surface area as measured using iTEM image analysis software (SIS) in ten fields of muscle sections from at least three animals for each group. Data are reported as mean \pm S.E.M. Statistical analysis was performed unpaired two-sided Student's $t$-test ( ${ }^{* *} P<0.001$ vs RD; ${ }^{\circ} P<0.05$ vs HFD). Scale bar $500 \mathrm{~nm}$. 
Table 4 Effects of in vivo testosterone treatment on mRNA expression of target genes in rabbit skeletal muscle.

\begin{tabular}{l}
\hline Gene \\
\hline Fiber types: \\
MYH1 \\
MYH2 \\
MYH4 \\
MYH7 \\
TBX15 \\
Mitochondrial oxidative function: \\
CS \\
NDUFB3 \\
NDUFB5 \\
NDUFS1 \\
SDHB \\
SLC25A12 \\
Atrophy, myogenesis and muscle differentiation: \\
FBXO32 \\
MYOG \\
MYF5 \\
PAX7 \\
ITGA7 \\
MYOD \\
GluCOSe, lipid and energy metabolism: \\
PPARA \\
IRS1 \\
LPL \\
UCP1 \\
UCP2 \\
UCP3
\end{tabular}

\begin{tabular}{c}
\hline RD \\
\hline $100.20(45.30-143.71)$ \\
$88.67(60.42-123.63)$ \\
$60.31(36.87-164.96)$ \\
$32.64(3.65-186.79)$ \\
$100.43(75.77-114.24)$
\end{tabular}

$88.11(68.18-129.37)$

$93.12(60.71-130.26)$

$95.81(69.86-122.31)$

$76.39(56.79-143.40)$

$84.91(59.00-140.35)$

$93.68(76.08-123.21)$

$96.03(51.46-124.81)$

$92.87(73.56-121.41)$

92.55 (70.76-129.42)

95.39 (58.55-129.28)

103.01 (85.64-119.82)

88.42 (55.59-131.58)

$91.40(64.15-137.61)$

$102.30(80.32-120.49)$

$88.99(60.41-142.45)$

$92.13(64.90-119.98)$

90.82 (55.16-135.27)

$92.20(53.01-129.61)$

\begin{tabular}{|c|c|}
\hline RD + testosterone & $P$ \\
\hline 90.93 (85.42-98.88) & 0.822 \\
\hline 132.43 (93.72-166.67) & 0.112 \\
\hline $102.96(70.37-207.14)$ & 0.379 \\
\hline 199.75 (50.30-353.00) & 0.223 \\
\hline 93.94 (87.23-147.09) & 0.763 \\
\hline 95.95 (85.99-134.47) & 0.510 \\
\hline $105.57(79.02-119.51)$ & 0.749 \\
\hline 117.18 (79.64-131.27) & 0.560 \\
\hline $100.92(85.39-104.88)$ & 0.533 \\
\hline $105.89(96.36-114.82)$ & 0.417 \\
\hline 107.37 (99.73-114.86) & 0.395 \\
\hline 116.80 (99.19-123.92) & 0.383 \\
\hline 136.88 (94.56-168.03) & 0.126 \\
\hline $95.26(81.81-130.72)$ & 0.704 \\
\hline 193.65 (138.14-222.75) & 0.009 \\
\hline $189.02(137.64-198.60)$ & 0.002 \\
\hline $98.16(79.95-126.81)$ & 0.778 \\
\hline $122.31(84.15-147.87)$ & 0.384 \\
\hline $152.17(100.43-163.82)$ & 0.124 \\
\hline $220.93(211.07-328.42)$ & $<0.001$ \\
\hline $102.72(71.49-111.62)$ & 0.836 \\
\hline $91.72(48.56-144.81)$ & 0.953 \\
\hline 86.68 (47.64-131.79) & 0.955 \\
\hline
\end{tabular}

The target genes mRNA expression was evaluated using semi-quantitative RT-PCR in skeletal muscle samples from RD $(n=15)$ and and RD + testosterone $(n=4)$ rabbits. Data were calculated according to the comparative $C_{t}$ method, using $18 \mathrm{~S}$ rRNA subunit as the reference gene for normalization. Results are expressed in percentage over RD and are reported as median and quartiles. Bold indicates genes with significantly different expression $(P<0.05)$ comparing RD vs RD + testosterone in the post hoc Mann-Whitney analysis.

$\mathrm{RD}+$ testosterone + PhyEx rabbits, we confirmed that the global fiber type composition and metabolism of skeletal muscle from RD animals was not significantly affected by testosterone supplementation (data not shown). As shown in Table 6, testosterone supplementation did not change any of the physical and metabolic parameters in RD animals either with or without PhyEx, when compared to their untreated counterpart, apart from the obvious changes in hormonal parameters (Table 6).

\section{Discussion}

Change in lifestyle, which includes the increase of physical activity, is an important and effective strategy to combat MetS-associated hypogonadism (Morelli et al. 2019). However, the ability of MetS subjects to perform physical activity might be suboptimal or even compromised, due to a detrimental effect of the syndrome on overall health and, in particular, on body composition. We here demonstrated that TTh in experimental MetS increases the capacity to perform physical exercise by improving skeletal muscle oxidative activity and ability to sustain endurance exercise. Indeed, physical activity is nowadays regarded as a major intervention for preventing or halting metabolic disturbances, such as those characterizing MetS (Bird \& Hawley 2017, Wewege et al. 2018). Skeletal muscle represents one of the most important tissues in maintaining normal whole-body metabolism and energy homeostasis because of its high contribution to the whole body mass and to its metabolic capacity (Zurlo et al. 1990). In an experimental (HFD-induced) MetS model associated with hypogonadism, we recently described a reduced ability of rabbits to perform physical exercise (Morelli et al. 2019). In the present study, we demonstrate that the reduced response to endurance training observed in MetS animals was associated with peculiar HFD-induced modifications in the skeletal muscle fiber composition and metabolism. More relevant, we herein report that when supplemented with testosterone, MetS rabbits exhibited skeletal muscle morphological and functional features similar to those observed in RD animals, including a normalized response 
D
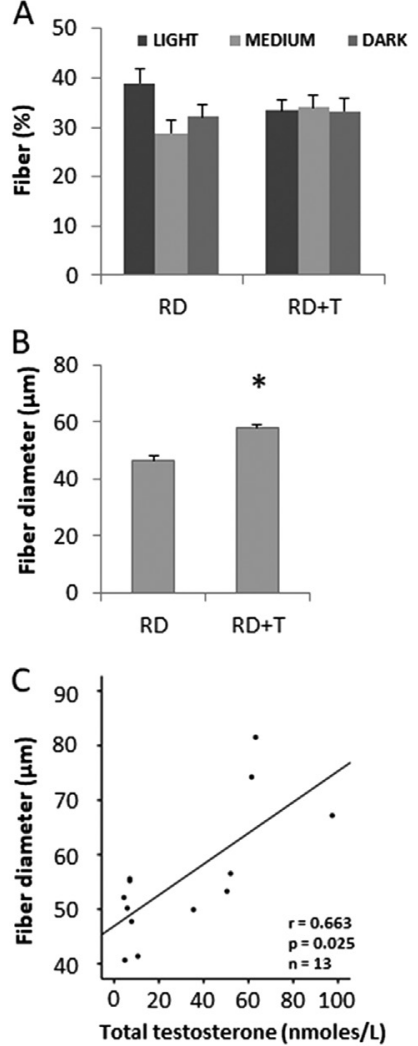
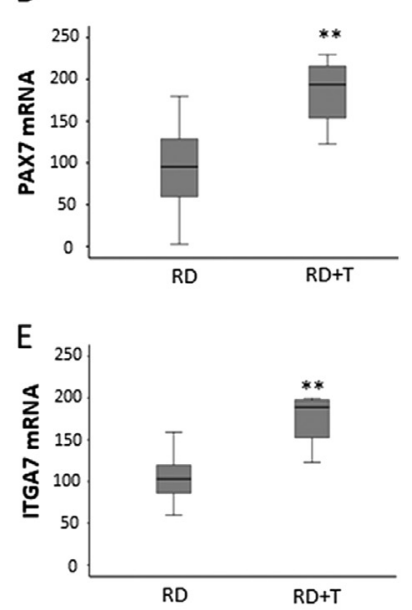

$\mathrm{F}$

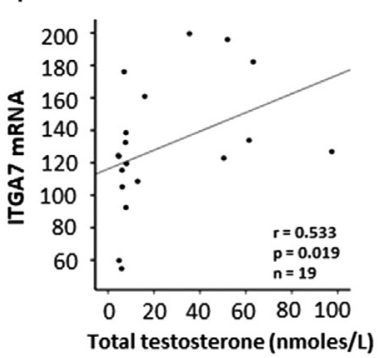

Figure 8

Effect of TTh on skeletal muscle composition and mRNA expression of regeneration/differentiation markers in RD group. (A) Quantification of light, medium and dark PAS-stained fibers calculated by counting ten fields from muscle sections of at least three animals for each experimental group. (B) Fiber diameter, as measured in muscle cross-sections of specimens from RD + testosterone group compared to $\mathrm{RD}$. Data were calculated by Image J $1.51 \mathrm{f}$ software in ten fields from at least three animals for each experimental group and expressed as mean \pm S.E.M. Statistical analysis between groups was performed with unpaired two-sided Student's $t$-test $(* P<0.001)$. (C) Relationship between fiber diameter and plasma levels of testosterone in the experimental groups, as derived from univariate Spearman's regression analysis. (D and E) TTh in RD rabbits significantly increased mRNA expression of the myogenesis-related gene PAX7 (D), and the muscle differentiation marker ITGA7/ $\alpha 7$ integrin (E). Data were calculated using the $2-\Delta \Delta \mathrm{Ct}$ comparative method, with the $18 \mathrm{~S}$ ribosomal RNA subunit used as reference gene for normalization, and are reported as mean \pm S.E.M. (RD, $n=15$;

$\mathrm{RD}+$ testosterone, $n=4)$. Statistical analysis was performed with KruskalWallis and post hoc Mann-Whitney non-parametric tests. ( $* P<0.001)$. (F) Relationship between ITGA7 mRNA and plasma levels of testosterone in the experimental groups, as derived from univariate Spearman's regression analysis.

to physical activity. On the other hand, testosterone supplementation on otherwise eugonadal rabbits (RD) did not result in significant changes in endurance ability, although increasing muscle fibers diameter. Given the well-known tight link existing between MetS and the development of hypogonadism in male population, our results add novel insights about the importance

of considering TTh as a favorable intervention for the efficacy of physical activity programs in MetS subjects, but not in eugonadal subjects (as it is in doping).

Skeletal muscle is one of the most highly plastic tissues, being capable of extensive remodeling in response to use, disuse and pathological perturbation. In particular, transformations of muscle fiber type may occur to facilitate functional adaptations for a muscle's specific needs, such as those requested during physical activity (Schiaffino \& Reggiani 2011). The functional adaptability of skeletal muscle depends upon the type of exercise: resistance exercise increases mass and strength, while endurance exercise can stimulate mitochondrial biogenesis and function, promoting aerobic metabolism. In this regard, endurance exercise has been found to be more effective in preventing cardiovascular and metabolic diseases (Shen et al. 2018).

In this study, we analyzed the vastus medialis muscle, a portion of the quadriceps femoris muscle group involved in knee extension, characterized by a mixed composition of type I/type II fibers and therefore useful for studying whether particular conditions could induce changes toward another fiber type. Histological evaluation of HFD muscle samples showed a reduction of slow/type I fibers, which are characterized by the use of oxidative metabolism and the ability to perform longlasting activities. On the contrary, we demonstrated an increased proportion of fast/glycolytic fibers, which rely on glycolytic metabolism and may not provide prolonged contractions. In fact, the PAS staining showed a reduction of light stained fibers and an increase of the medium/ dark stained ones, more rich of glycogen, the substrate of anaerobic contractions. Moreover, HFD induced the expression of the type IIB glycolytic fiber markers MYH4 (Schiaffino \& Reggiani 2011) and of the mesodermal transcription factor testosterone-box 15 (TBX15), a highly and specifically expressed transcription factor in glycolytic fast-twitch myofibers (Lee et al. 2015). Interestingly, TBX15 ablation determines a shift to oxidative myofibers (Lee et al. 2015). The concomitant reduced expression of the type I marker MYH7 in vastus medialis from HFD rabbits further demonstrated a morphofunctional shift in terms of both fiber composition and contractile properties. Interestingly, the aforementioned fiber type modifications were accompanied by muscle atrophy and by an impairment of myogenic capacity, as demonstrated by the reduction of fiber diameter, by the increase of the atrophy-related gene FBX032 and by the reduction of myogenin gene expression in HFD muscles. Overall, these observations are consistent with previous findings 
from human (Stuart et al. 2013) and animal (Denies et al. 2014) studies, showing that MetS condition, in particular insulin resistance, correlates with a higher content of glycolytic fibers and with a loss of the oxidative ones. In agreement with all these muscle structural changes, HFD animals showed a reduced physical performance in response to endurance training. Indeed, the HFD-induced new asset of muscle composition may account for the impairment of exercise performance, since the muscular fatigue resistance during endurance training exercises is based on the integrity and activity of slow type I/oxidative fibers. Interestingly, we found that the reduction of muscular oxidative metabolism was exalted by physical exercise with a further shift to a glycolytic phenotype, thus suggesting the incapability of HFD muscle of working through oxidative fibers that instead should be normally induced by the endurance training (Pette 2002, Shen et al. 2018).

In this regard, our results obtained in the testosteronetreated HFD group are of particular relevance for two reasons: (1) they demonstrate that testosterone was able to counteract all the HFD-induced changes in the skeletal muscle fiber composition of untrained rabbits, maintaining a glycolytic/oxidative fiber ratio similar to that of RD group; (2) they suggest that the maintenance of a 'RD-like' skeletal muscle composition by testosterone treatment allowed a normalization in the response to physical activity.

It is well known that testosterone exerts trophic effects on muscle, mainly promoting protein synthesis, fiber hypertrophy and satellite cells/myonuclei proliferation and differentiation (Herbst \& Bhasin 2004). In fact, TTh is regarded an effective approach to increase muscle mass and strength in both young and old men with androgen deficiency (Bhasin et al. 1997, Bhasin et al. 2018). By using a murine model of aging-associated sarcopenia, TTh significantly enhanced muscle growth, while increasing muscle mass and hypertrophy of both types I and II fibers and preventing age-related increase in oxidative stress and muscle cell apoptosis (Kovacheva et al. 2010).
Moreover, TTh also reduced orchiectomy-induced expression of several atrophy-related markers in skeletal muscle (White et al. 2013). In line with these anabolic and anti-catabolic effects of TTh, present data show a positive effect of testosterone on HFD-induced atrophy and on the expression of myogenic (MYOG, MYF5, PAX7) and differentiation (MYOD and ITGA7) genes that was also positively associated with testosterone plasma levels. Accordingly, an increase of PAX7 immunopositivity and the presence of newly generated MYH3-positive fibers were detected in skeletal muscle from testosterone-treated HFD rabbits. Noteworthy, we here demonstrate for the first time that testosterone normalized the MetS-induced shift of type I/II fibers, as demonstrated by PAS staining and $\mathrm{MYH} / \mathrm{Tbx} 15$ gene expression, thereby promoting a muscle asset indicative of predominant oxidative metabolism, even in untrained rabbits. Accordingly, we found that the expression of mitochondrial enzymes crucial for cell respiration was not only significantly increased in skeletal muscle from testosterone-treated HFD rabbits, but also positively correlated to testosterone plasma levels. Moreover, ultrastructural analysis of muscle samples showed that testosterone was able to maintain mitochondrial integrity counteracting HFD-induced degeneration of internal membrane cristae. Similar results were found also in terms of both ATP production, as marker of mitochondrial respiration, and in terms of mitochondrial mass, as evaluated by TOM20 protein expression. Noteworthy, mitochondrial dysfunction is crucially contributing to the complex etiology of sarcopenia even in the context of metabolic diseases (Coen et al. 2018). Treatment in male mice increases the expression of genes involved in mitochondrial biogenesis and heat production in skeletal muscle via an AR-dependent pathway (Usui et al. 2014). In addition, in men, testosterone correlates with genetic and functional markers of mitochondrial function in skeletal muscle (Pitteloud et al. 2005). Therefore, the positive effects of testosterone treatment in MetS animals on fatigue resistant fibers and mitochondrial integrity - substantiated by a

Table 5 Effects of physical exercise in testosterone-treated RD rabbits.

Running distance $(\mathrm{m})$
Running time $(\mathrm{s})$
Lactate (nmol/L)
Lactate/running distance (nmol/L for each $100 \mathrm{~m})$

\begin{tabular}{c}
\hline RD + testosterone + PhyEx $(n=4)$ \\
\hline $204.00(189.30 ; 218.70)$ \\
$612.00(567.90 ; 656.10)$ \\
Pre-exercise: $2.70(0.86 ; 4.54)$ \\
End: $17.2(2.87 ; 31.53)$ \\
$8.59(0.95 ; 16.23)$
\end{tabular}

\begin{tabular}{c}
\hline P vs RD + PhyEx \\
\hline 0.839 \\
0.839 \\
0.374 \\
0.839 \\
0.8393
\end{tabular}

Measurements were performed before starting (pre-exercise) and at the end of the exercise (end). The $P$ value is vs the relative RD group of Table 3 , untreated with testosterone. Data are reported as median with 95\% confidence intervals (in brackets).

https://joe.bioscientifica.com https://doi.org/10.1530/JOE-19-0532 (c) 2020 Society for Endocrinology Published by Bioscientifica Ltd. Printed in Great Britain 
Table 6 Metabolic parameters of testosterone-treated RD rabbits with and without PhyEx.

\begin{tabular}{l}
\hline \\
\hline Total body weight $(\mathrm{kg})$ \\
Blood glucose $(\mathrm{g} / \mathrm{L})$ \\
OGTT (iAUC) \\
Cholesterol (mg/dL) \\
Triglycerides (mg/dL) \\
MAP (mmHg) \\
VAT (g/kg of total weight) \\
Testosterone (nmol/L) \\
LH (ng/mL)
\end{tabular}

\begin{tabular}{c}
\hline RD + testosterone $(n=4)$ \\
\hline $3.75(3.63-4.18)$ \\
$0.97(0.86-1.08)$ \\
$141.70(130.12-176.07)$ \\
$49.00(48.25-49.75)$ \\
$124.00(88.50-149.00)$ \\
$78.43(65.00-96.09)$ \\
$8.98(7.43-9.85)$ \\
$51.20(39.18-60.40)$ \\
$0.05(0.02-0.09)$ \\
\hline
\end{tabular}

\begin{tabular}{c}
\hline P vs RD \\
\hline 0.885 \\
0.262 \\
0.307 \\
0.665 \\
0.062 \\
0.810 \\
0.961 \\
0.001 \\
0.014 \\
\hline
\end{tabular}

\begin{tabular}{c}
\hline RD + testosterone + PhyEx $(n=4)$ \\
\hline $3.72(3.59-3.84)$ \\
$0.93(0.81-0.98)$ \\
$147.50(137.67-155.30)$ \\
$49.50(48.25-50.00)$ \\
$75.00(67.50-87.00)$ \\
$84.00(80.00-88.00)$ \\
$8.50(7.66-9.35)$ \\
$71.60(55.40-93.50)$ \\
$0.06(0.02-0.07)$ \\
\hline
\end{tabular}

\begin{tabular}{c}
\hline P vs RD + PhyEx \\
\hline 0.454 \\
0.304 \\
0.304 \\
0.773 \\
0.142 \\
0.414 \\
0.454 \\
0.003 \\
0.014
\end{tabular}

Results are reported as medians with quartiles in brackets. The p value is vs the relative group of Table 1, untreated with testosterone.

significant improvement in the exercise performance and by the reduced lactate production - might be one of the major underlying mechanisms of TTh-induced metabolic improvements.

Altered glucose and lipid metabolism in HFD rabbits could thus be the cause of skeletal muscle modifications. Hence, we speculate that the amelioration of some MetS parameters (in particular fasting glucose levels, glucose tolerance and visceral adipose tissue accumulation) upon testosterone treatment could indirectly contribute to the normalization and/or improvement in skeletal muscle feature. Consistent with this hypothesis, we also found that TTh counteracted HFD-induced i.m. lipid accumulation. Recent studies demonstrated that a western-like diet may induce changes in skeletal muscle metabolism that promote lipid utilization over glucose oxidation as substrates of mitochondrial respiration (McCurdy et al. 2016, GonzalezArmenta et al. 2019). These changes were associated to a decrease in oxidative capacity and an impairment of glucose metabolism. Moreover, measures of mitochondrial respiration in response to fatty acids were significantly and positively correlated with insulin resistance (GonzalezArmenta et al. 2019) We did not analyze specific effects of HFD, with and without testosterone administration, on mitochondrial bioenergetics. However, our results, concerning HFD-induced and testosterone-counteracted skeletal muscle changes - including mitochondrial structural alterations, lipid accumulation, reduced content of oxidative fibers, as well as reduced oxidative metabolism - suggest that all these changes are associated to the diet composition. In addition, our results indicate that testosterone treatment counteracted these HFD-induced muscle changes, most likely facilitating muscle glucose uptake and oxidative metabolism, and this could be related to the maintenance of an intact mitochondrial structure.

In physiological conditions, blood glucose, plasmafree fatty acids, muscle glycogen, and i.m. triglycerides are the major substrate sources for energy production in skeletal muscles. In particular, during exercise, blood glucose level can be increased primarily by means of an augmented glycogenolysis in the liver (Brooks et al. 2005). On the contrary, impairment of blood glucose homeostasis under MetS condition is associated with insulin resistance and altered glucose uptake by skeletal muscle. Accordingly, in RD + PhyEx, at the end of exercise, glycemia tended to increase, whereas in HFD + PhyEx rabbits the high glycemic levels remained unchanged. TTh partially reduced basal glycemia and allowed a sustained increase in glycemia upon PhyEx, thus suggesting a restoration of the glucose mobilization as important source of substrate for exercising muscle. However, based on our results we cannot rule out that metabolic improvements following TTh may be a consequence of positive direct effects of testosterone on skeletal muscle ability in metabolizing glucose and lipids. In this regard, we observed an up-regulation of IRS1 gene in the vastus medialis of testosterone-treated rabbits, in line with a previous study that obtained the same results on castrated rats treated with testosterone ( $\mathrm{Wu}$ et al. 2010). Similarly, we report a positive effect of testosterone on the expression of genes involved in regulating lipid metabolism in skeletal muscle, such as PPAR $\alpha$, LPL and UCPs. In human skeletal muscle, expression of PPAR $\alpha$ and of other genes regulating lipid metabolism, in particular LPL, are tightly related (Zhang et al. 2004). LPL activity, especially in oxidative muscle, is normally high to allow the release of free fatty acids and their subsequent uptake by myofibers, for energy production. UCPs are proteins located in the mitochondrial inner membrane with the function to uncouple the oxidation of fuels, mainly fatty acids, from ATP production, so the energy associated with oxidation is dissipated as heat (Bouillaud et al. 2016). Mitochondrial uncoupling and the consequent energy dissipation are of great importance in relation 
to obesity, diabetes, and MetS (Li et al. 2018). UCP1 is highly expressed in brown adipose tissue with thermogenic function. However, an ectopic expression of UCP1 in the skeletal muscle of transgenic mice is associated with an increase of whole-body energy expenditure, resulting in the restoration of insulinemia, cholesterolemia and adiposity, and a decrease of type IIB fiber content (Li et al. 2000, Couplan et al. 2002). Moreover, UCP1 expression in the skeletal muscle of transgenic mice protects them from HFD-induced obesity-associated disturbances of glucose homeostasis (Katterle et al. 2008). UCP2 and UCP3 are broadly expressed UCP isoforms and their expression is upregulated after starvation and correlates with the use of fatty acids as mitochondrial substrates, suggesting a novel role, different from the uncoupling one (Bouillaud et al. 2016).

Generation of genetically modified knock-out (KO) mouse models in which the androgen receptor (AR) has been deleted either globally or in a tissueand/or cell-specific manner gave also important insights into the mechanism by which androgens act on the musculoskeletal system. In global-ARKOs muscle mass was decreased by up to $20 \%$ compared with wild-type males (MacLean et al. 2008, Ophoff et al. 2009). However, in contrast with our observations, ARKO mice showed less fast-twitch (type II) and reduced contractile strength accompanied by an increase in the content of slow-twitch (type I) fibers (MacLean et al. 2008, Ophoff et al. 2009). The discrepancy indicates that the metabolic background may interfere with the testosterone actions on skeletal muscles as expected only considering the classical AR-mediated pathway. Moreover, data from several muscle-specific ARKOs, including those targeting satellite cells, myocytes, myoblasts, and/or myofibers, provide evidence for a partial direct role of androgens via the AR in skeletal muscle to regulate its mass (Chambon et al. 2010, Rana et al. 2016). So it seems that other sites of action of testosterone might contribute in mediating the anabolic effects of testosterone on skeletal muscle mass. Indeed, a neuron-ARKO mouse model in which the AR is deleted specifically in neurons in the brain clearly demonstrated that brain AR acts to positively regulate hind-limb mass (Davey et al. 2017).

The most intriguing finding of our study is the demonstration that treating HFD rabbits with testosterone was able to allow a normal exercise capacity that was impaired before. This is tantamount to say that a testosterone supplementation might help MetS subjects to be able to perform physical exercise. Interestingly, when we treated with testosterone an additional group of RD rabbits with normal level of testosterone, we did not observe any significant effects either on muscle fiber composition and metabolism or in terms of response to physical activity. We only evidenced a trophic effect, as demonstrated by an increased fiber diameter, as well as by an induction of some genes related to myogenesis, including PAX7 and ITGA7. Accordingly, testosterone plasma levels was positively associated with fiber diameter and ITGA7 mRNA expression in RD group, further supporting the view of a trophic effect of testosterone independently of HFD. Consistent with our findings, it has been demonstrated that testosterone administration in supraphysiological doses to healthy eugonadal men is associated with significant increases in muscle size due to muscle fiber hypertrophy without changing the relative proportions of type I and type II fibers (SinhaHikim et al. 2002). Overall, testosterone treatment in RD rabbits did not change the global metabolic conditions and affected the skeletal muscle features at a little extent, if compared to that observed in testosterone-treated HFD group. Hence, we can hypothesize that the positive effects of testosterone observed in the HFD group are linked to HFD-induced features, including testosterone deficiency, and not a mere androgenic effect, thus further supporting the use of TTh in pathological conditions characterized by hypogonadism but not in eugonadal subjects.

A recent meta-analysis of intervention studies in hypogonadal patients with metabolic derangements reported that combination of testosterone supplementation and lifestyle modifications was able to improve, more than lifestyle change itself, body composition (reduction of fat mass and increase in lean mass), leading to an amelioration of lipid profile and insulin resistance (Corona et al. 2015). Whether or not TTh could ameliorate exercise performance in MetS remains to be investigated. In fact, the available studies on the effect of testosterone supplementation on muscle strength and function were mainly conducted in older men and results are controversial. In a RCT, which compared older healthy men with low-to-normal testosterone levels receiving TTh or placebo for 3 years, a modest but significant improvement in muscle mass and power was shown in the active arm (Storer et al. 2017). Accordingly, in the 'Testosterone Trials', a coordinated set of seven placebo-controlled, double-blind trials aimed at determining the efficacy of increasing testosterone levels of older men with androgen deficiency, a small benefit was reported with respect to walking performance in hypogonadal patients following 12 weeks of testosterone therapy (Snyder et al. 2018). 
However, this improvement was not confirmed when only older hypogonadal men with limited mobility were considered (Snyder et al. 2016). Conversely, in a trial involving more than 200 elderly hypogonadal men with limitations in mobility randomly treated with TTh or placebo for 9 months, a significant improvement in legand chest-press strength as well as in stair-climbing power was associated with TTh (Basaria et al. 2010). A recent reanalysis of systematic reviews and meta-analyses on the topic suggests that, in older men with clinical muscle weakness and low testosterone levels, treatment can be justified in daily clinical practice to improve muscle mass, muscle strength and/or physical performance (De Spiegeleer et al. 2018).

In conclusion, in an animal model of MetS, associated with hypogonadism, TTh showed a positive effect not only in ameliorating the glycometabolic profile, but also in modifying the morpho-functional features of skeletal muscles. Indeed, following testosterone treatment, HFD muscles were almost comparable to $\mathrm{RD}$ muscles. In fact, fiber muscle composition, mitochondria features and SDH activity were all improved and shifted toward an oxidative metabolism, instead of a glycolytic one. All the observed muscle modifications resulted in a better exercise performance. Accordingly, in aged male mice, testosterone administration, along with a low-intensity physical training, improved skeletal muscle mitochondrial biogenesis/quality and physical performance (Guo et al. 2012). Adequate clinical studies are required to confirm also in humans results obtained in these animal models. Our findings support the use of TTh in MetS as an intervention to favor lifestyle correction and, in particular, the adherence to physical exercise programs. As soon as lifestyle changes are established and could orchestrate their favorable effects (Morelli et al. 2019) the need for further testosterone supplementation can be reconsidered. Finally, our data do not suggest the use of testosterone administration to otherwise eugonadal individuals to improve aerobic endurance performances.

\section{Declaration of interest}

The authors declare that there is no conflict of interest that could be perceived as prejudicing the impartiality of the research reported.

\section{Funding}

This work did not receive any specific grant from any funding agency in the public, commercial, or not-for-profit sector.

\section{References}

Abrigo J, Rivera JC, Aravena J, Cabrera D, Simon F, Ezquer F, Ezquer M \& Cabello-Verrugio C 2016 High fat diet-induced skeletal muscle wasting is decreased by Mesenchymal Stem Cells Administration: implications on oxidative stress, ubiquitin proteasome pathway activation, and myonuclear apoptosis. Oxidative Medicine and Cellular Longevity 2016 9047821. (https://doi.org/10.1155/2016/9047821)

Basaria S, Coviello AD, Travison TG, Storer TW, Farwell WR, Jette AM, Eder R, Tennstedt S, Ulloor J, Zhang A, et al. 2010 Adverse events associated with testosterone administration. New England Journal of Medicine 363 109-122. (https://doi.org/10.1056/NEJMoa1000485)

Baumgartner RN, Waters DL, Gallagher D, Morley JE \& Garry PJ 1999 Predictors of skeletal muscle mass in elderly men and women. Mechanisms of Ageing and Development 107 123-136. (https://doi. org/10.1016/s0047-6374(98)00130-4)

Bhasin S, Storer TW, Berman N, Yarasheski KE, Clevenger B, Phillips J, Lee WP, Bunnell TJ \& Casaburi R 1997 Testosterone replacement increases fat-free mass and muscle size in hypogonadal men. Journal of Clinical Endocrinology and Metabolism 82 407-413. (https://doi. org/10.1210/jcem.82.2.3733)

Bhasin S, Storer TW, Singh AB, Woodhouse L, Singh R, Artaza J, Taylor WE, Sinha-Hikim I, Jasuja R \& Gonzalez-Cadavid N 2004 Testosterone effects on the skeletal muscle. In Testosterone: Action, Deficiency, Substitution, 3rd ed., pp. 255-282. Cambridge University Press.

Bhasin S, Brito JP, Cunningham GR, Hayes FJ, Hodis HN, Matsumoto AM, Snyder PJ, Swerdloff RS, Wu FC \& Yialamas MA 2018 Testosterone therapy in men with androgen deficiency syndromes: an Endocrine Society clinical practice guideline. Journal of Clinical Endocrinology and Metabolism 103 1715-1744. (https://doi.org/10.1210/jc.2018-00229)

Bhatt BA, Dube JJ, Dedousis N, Reider JA \& O'Doherty RM 2006 Dietinduced obesity and acute hyperlipidemia reduce IкB $\alpha$ levels in rat skeletal muscle in a fiber-type dependent manner. American Journal of Physiology: Regulatory, Integrative and Comparative Physiology 290 R233-R240. (https://doi.org/10.1152/ajpregu.00097.2005)

Bird SR \& Hawley JA 2017 Update on the effects of physical activity on insulin sensitivity in humans. BMJ Open Sport and Exercise Medicine 21 e000143. (https://doi.org/10.1136/bmjsem-2016-000143)

Bouillaud F, Alves-Guerra MC \& Ricquier D 2016 UCPs, at the interface between bioenergetics and metabolism. Biochimica et Biophysica Acta 1863 2443-2456. (https://doi.org/10.1016/j.bbamcr.2016.04.013)

Brooks GA, Fahey TD \& Baldwin KM 2005 Exercise Physiology: Human Bioenergetics and Its Applications. New York: McGraw-Hill.

Cangiano B, Duminuco P, Vezzoli V, Guizzardi F, Chiodini I, Corona G, Maggi M, Persani L \& Bonomi M 2019 Evidence for a common genetic origin of classic and milder adult-onset forms of isolated hypogonadotropic hypogonadism. Journal of Clinical Medicine 8 E126. (https://doi.org/10.3390/jcm8010126)

Carson JA \& Manolagas SC 2015 Effects of sex steroids on bones and muscles: similarities, parallels, and putative interactions in health and disease. Bone 80 67-78. (https://doi.org/10.1016/j.bone.2015.04.015)

Chambon C, Duteil D, Vignaud A, Ferry A, Messaddeq N, Malivindi R, Kato S, Chambon P \& Metzger D 2010 Myocytic androgen receptor controls the strength but not the mass of limb muscles. PNAS 107 14327-14332. (https://doi.org/10.1073/pnas.1009536107)

Church T 2011 Exercise in obesity, metabolic syndrome, and diabetes. Progress in Cardiovascular Diseases 53 412-418. (https://doi. org/10.1016/j.pcad.2011.03.013)

Coen PM, Musci RV, Hinkley JM \& Miller BF 2018 Mitochondria as a target for mitigating sarcopenia. Frontiers in Physiology 91883. (https://doi.org/10.3389/fphys.2018.01883)

Corona G, Vignozzi L, Sforza A, Mannucci E \& Maggi M 2015 Obesity and late-onset hypogonadism. Molecular and Cellular Endocrinology 418 120-133. (https://doi.org/10.1016/j.mce.2015.06.031)

Corona G, Giagulli VA, Maseroli E, Vignozzi L, Aversa A, Zitzmann M, Saad F, Mannucci E \& Maggi M 2016 THERAPY OF ENDOCRINE https://joe.bioscientifica.com https://doi.org/10.1530/JOE-19-0532 (c) 2020 Society for Endocrinology Published by Bioscientifica Ltd. 
DISEASE: Testosterone supplementation and body composition: results from a meta-analysis study. European Journal of Endocrinology 174 R99-R116. (https://doi.org/10.1530/EJE-15-0262)

Couplan E, Gelly C, Goubern M, Fleury C, Quesson B, Silberberg M, Thiaudiere E, Mateo P, Lonchampt M, Levens N, et al. 2002 High level of uncoupling protein 1 expression in muscle of transgenic mice selectively affects muscles at rest and decreases their IIb fiber content. Journal of Biological Chemistry 277 43079-43088. (https://doi. org/10.1074/jbc.M206726200)

Davey RA, Clarke MV, Russell PK, Rana K, Seto J, Roeszler KN, How JMY, Chia LY, North K \& Zajac JD 2017 Androgen action via the androgen receptor in neurons within the brain positively regulates muscle mass in male mice. Endocrinology 158 3684-3695. (https://doi.org/10.1210/ en.2017-00470)

De Spiegeleer A, Beckwée D, Bautmans I, Petrovic M \& Sarcopenia Guidelines Development group of the Belgian Society of Gerontology and Geriatrics (BSGG) 2018 Pharmacological interventions to improve muscle mass, muscle strength and physical performance in older people: an umbrella review of systematic reviews and metaanalyses. Drugs and Aging 35 719-734. (https://doi.org/10.1007/ s40266-018-0566-y)

Denies MS, Johnson J, Maliphol AB, Bruno M, Kim A, Rizvi A, Rustici K \& Medler S 2014 Diet-induced obesity alters skeletal muscle fiber types of male but not female mice. Physiological Reports 2 e00204. (https:// doi.org/10.1002/phy2.204)

Filippi S, Vignozzi L, Morelli A, Chavalmane AK, Sarchielli E, Fibbi B, Saad F, Sandner P, Ruggiano P, Vannelli GB, et al. 2009 Testosterone partially ameliorates metabolic profile and erectile responsiveness to PDE5 inhibitors in an animal model of male metabolic syndrome. Journal of Sexual Medicine 6 3274-3288. (https://doi.org/10.1111/ j.1743-6109.2009.01467.x)

Finkelstein JS, Lee H, Burnett-Bowie SA, Pallais JC, Yu EW, Borges LF, Jones BF, Barry CV, Wulczyn KE, Thomas BJ, et al. 2013 Gonadal steroids and body composition, strength, and sexual function in men. New England Journal of Medicine 369 1011-1022. (https://doi. org/10.1056/NEJMoa1206168)

Gonzalez BD, Jim HSL, Small BJ, Sutton SK, Fishman MN, Zachariah B, Heysek RV \& Jacobsen PB 2016 Changes in physical functioning and muscle strength in men receiving androgen deprivation therapy for prostate cancer: a controlled comparison. Supportive Care in Cancer $\mathbf{2 4}$ 2201-2207. (https://doi.org/10.1007/s00520-015-3016-y)

Gonzalez-Armenta JL, Gao Z, Appt SE, Vitolins MZ, Michalson KT, Register TC, Shively CA \& Molina AJA 2019 Skeletal muscle mitochondrial respiration is elevated in female cynomolgus macaques fed a western compared with a Mediterranean diet. Journal of Nutrition 149 1493-1502. (https://doi.org/10.1093/jn/nxz092)

Grinspoon S, Corcoran C, Lee K, Burrows B, Hubbard J, Katznelson L, Walsh M, Guccione A, Cannan J, Heller H, et al. 1996 Loss of lean body and muscle mass correlates with androgen levels in hypogonadal men with acquired immunodeficiency syndrome and wasting. Journal of Clinical Endocrinology and Metabolism $\mathbf{8 1}$ 4051-4058. (https://doi.org/10.1210/jcem.81.11.8923860)

Guo W, Wong S, Li M, Liang W, Liesa M, Serra C, Jasuja R, Bartke A, Kirkland JL, Shirihai O, et al. 2012 Testosterone plus low-intensity physical training in late life improves functional performance, skeletal muscle mitochondrial biogenesis, and mitochondrial quality control in male mice. PLOS ONE 7 e51180. (https://doi.org/10.1371/ journal.pone.0051180)

Herbst KL \& Bhasin S 2004 Testosterone action on skeletal muscle. Current Opinion in Clinical Nutrition and Metabolic Care 7 271-277. (https://doi.org/10.1097/00075197-200405000-00006)

Hernandez N, Torres SH, Vera O, De Sanctis JB \& Flores E 2001 Muscle fiber composition and capillarization in relation to metabolic alterations in hypertensive men. Journal of Medicine 32 67-82.

Ingle L, Mellis M, Brodie D \& Sandercock GR 2017 Associations between cardiorespiratory fitness and the metabolic syndrome in British men. Heart 103 524-528. (https://doi.org/10.1136/ heartjnl-2016-310142)

Katterle Y, Keipert S, Hof J \& Klaus S 2008 Dissociation of obesity and insulin resistance in transgenic mice with skeletal muscle expression of uncoupling protein 1. Physiological Genomics 32 352-359. (https:// doi.org/10.1152/physiolgenomics.00194.2007)

Kovacheva EL, Hikim AP, Shen R, Sinha I \& Sinha-Hikim I 2010 Testosterone supplementation reverses sarcopenia in aging through regulation of myostatin, c-Jun NH2-terminal kinase, Notch, and Akt signaling pathways. Endocrinology 151 628-638. (https://doi. org/10.1210/en.2009-1177)

Lakka TA \& Laaksonen DE 2007 Physical activity in prevention and treatment of the metabolic syndrome. Applied Physiology, Nutrition, and Metabolism 32 76-88. (https://doi.org/10.1139/h06-113)

Lee SR, Khamoui AV, Jo E, Park BS, Zourdos MC, Panton LB, Ormsbee MJ \& Kim JS 2015 Effects of chronic high fat feeding on skeletal muscle mass and function in middle-aged mice. Aging Clinical and Experimental Research 27 403-411. (https://doi.org/10.1007/s40520-015-0316-5)

Li B, Nolte LA, Ju JS, Han DH, Coleman T, Holloszy JO \& Semenkovich CF 2000 Skeletal muscle respiratory uncoupling prevents diet-induced obesity and insulin resistance in mice. Nature Medicine 6 1115-1120. (https://doi.org/10.1038/80450)

Li S, Zhou X, Jo E, Mahzari A, Fouda S, Li D, Zhang K \& Ye JM 2018 Preconditioning lessens high fat induced metabolic syndrome along with markers of increased metabolic capacity in muscle and adipose tissue. Bioscience Reports 38 BSR20181873. (https://doi.org/10.1042/ BSR20181873)

MacLean HE, Chiu WS, Notini AJ, Axell AM, Davey RA, McManus JF, Ma C, Plant DR, Lynch GS \& Zajac JD 2008 Impaired skeletal muscle development and function in male, but not female, genomic androgen receptor knockout mice. FASEB Journal 22 2676-2689. (https://doi.org/10.1096/fj.08-105726)

Maneschi E, Morelli A, Filippi S, Cellai I, Comeglio P, Mazzanti B, Mello T, Calcagno A, Sarchielli E, Vignozzi L, et al. 2012 Testosterone treatment improves metabolic syndrome-induced adipose tissue derangements. Journal of Endocrinology 215 347-362. (https://doi. org/10.1530/JOE-12-0333)

McCurdy CE, Schenk S, Hetrick B, Houck J, Drew BG, Kaye S, Lashbrook M, Bergman BC, Takahashi DL, Dean TA, et al. 2016 Maternal obesity reduces oxidative capacity in fetal skeletal muscle of Japanese macaques. JCI Insight 1 e86612. (https://doi.org/10.1172/jci. insight.86612)

Morelli A, Vignozzi L \& Maggi M 2016 Hypogonadotropic hypogonadism and metabolic syndrome: insights from the high-fat diet experimental rabbit animal model. Minerva Endocrinologica 41 240-249.

Morelli A, Filippi S, Comeglio P, Sarchielli E, Cellai I, Pallecchi M, Bartolucci G, Danza G, Rastrelli G, Corno C, et al. 2019 Physical activity counteracts metabolic syndrome-induced hypogonadotropic hypogonadism and erectile dysfunction in the rabbit. American Journal of Physiology: Endocrinology and Metabolism 316 E519-E535. (https://doi.org/10.1152/ajpendo.00377.2018)

Morley JE, Kaiser FE, Perry HM, Patrick P, Morley PM, Stauber PM, Vellas B, Baumgartner RN \& Garry PJ 1997 Longitudinal changes in testosterone, luteinizing hormone, and follicle stimulating hormone in healthy older men. Metabolism: Clinical and Experimental 46 410-413. (https://doi.org/10.1016/s0026-0495(97)90057-3)

Ophoff J, Van Proeyen K, Callewaert F, De Gendt K, De Bock K, Vanden Bosch A, Verhoeven G, Hespel P \& Vanderschueren D 2009 Androgen signaling in myocytes contributes to the maintenance of muscle mass and fiber type regulation but not to muscle strength or fatigue. Endocrinology 150 3558-3566. (https://doi.org/10.1210/en.20081509)

Pedersen BK \& Saltin B 2015 Exercise as medicine - evidence for prescribing exercise as therapy in 26 different chronic diseases. Scandinavian Journal of Medicine and Science in Sports 25 (Supplement 3) 1-72. (https://doi.org/10.1111/sms.12581) https://joe.bioscientifica.com https://doi.org/10.1530/JOE-19-0532 (c) 2020 Society for Endocrinology Published by Bioscientifica Ltd. Printed in Great Britain 
Pette D 2002 The adaptive potential of skeletal muscle fibers. Canadian Journal of Applied Physiology 27 423-448. (https://doi.org/10.1139/ h02-023)

Pitteloud N, Mootha VK, Dwyer AA, Hardin M, Lee H, Eriksson KF, Tripathy D, Yialamas M, Groop L, Elahi D, et al. 2005 Relationship between testosterone levels, insulin sensitivity, and mitochondrial function in men. Diabetes Care 28 1636-1642. (https://doi. org/10.2337/diacare.28.7.1636)

Rana K, Chiu MW, Russell PK, Skinner JP, Lee NK, Fam BC, Zajac JD \& MacLean HE 2016 Muscle-specific androgen receptor deletion shows limited actions in myoblasts but not in myofibers in different muscles in vivo. Journal of Molecular Endocrinology 57 125-138. (https://doi. org/10.1530/JME-15-0320)

Rapizzi E, Fucci R, Giannoni E, Canu L, Richter S, Cirri P \& Mannelli M 2015 Role of microenvironment on neuroblastoma SK-N-AS SDHB silenced cell metabolism and function. Endocrine-Related Cancer 22 409-417. (https://doi.org/10.1530/ERC-14-0479)

Roberts CK \& Barnard RJ 2005 Effects of exercise and diet on chronic disease. Journal of Applied Physiology 98 3-30. (https://doi. org/10.1152/japplphysiol.00852.2004)

Roy TA, Blackman MR, Harman SM, Tobin JD, Schrager M \& Metter EJ 2002 Interrelationships of serum testosterone and free testosterone index with FFM and strength in aging men. American Journal of Physiology: Endocrinology and Metabolism 283 E284-E294. (https://doi. org/10.1152/ajpendo.00334.2001)

Schiaffino S \& Reggiani C 2011 Fiber types in mammalian skeletal muscles. Physiological Reviews 91 1447-1531. (https://doi.org/10.1152/ physrev.00031.2010)

Shen L, Meng X, Zhang Z \& Wang T 2018 Physical exercise for muscle atrophy. Advances in Experimental Medicine and Biology 1088 529-545. (https://doi.org/10.1007/978-981-13-1435-3_24)

Sinha-Hikim I, Artaza J, Woodhouse L, Gonzalez-Cadavid N, Singh AB, Lee MI, Storer TW, Casaburi R, Shen R \& Bhasin S 2002 Testosteroneinduced increase in muscle size in healthy young men is associated with muscle fiber hypertrophy. American Journal of Physiology: Endocrinology and Metabolism 283 E154-E164. (https://doi. org/10.1152/ajpendo.00502.2001)

Sishi B, Loos B, Ellis B, Smith W, Du Toit EF \& Engelbrecht AM 2011 Diet-induced obesity alters signalling pathways and induces atrophy and apoptosis in skeletal muscle in a prediabetic rat model. Experimental Physiology 96 179-193. (https://doi.org/10.1113/ expphysiol.2010.054189)

Snyder PJ, Bhasin S, Cunningham GR, Matsumoto AM, StephensShields AJ, Cauley JA, Gill TM, Barrett-Connor E, Swerdloff RS,
Wang C, et al. 2016 Effects of testosterone treatment in older men. New England Journal of Medicine 374 611-624. (https://doi. org/10.1056/NEJMoa1506119)

Snyder PJ, Bhasin S, Cunningham GR, Matsumoto AM, StephensShields AJ, Cauley JA, Gill TM, Barrett-Connor E, Swerdloff RS, Wang C, et al. 2018 Lessons from the testosterone trials. Endocrine Reviews 39 369-386. (https://doi.org/10.1210/er.2017-00234)

Storer TW, Basaria S, Traustadottir T, Harman SM, Pencina K, Li Z, Travison TG, Miciek R, Tsitouras P, Hally K, et al. 2017 Effects of testosterone supplementation for 3 years on muscle performance and physical function in older men. Journal of Clinical Endocrinology and Metabolism 102 583-593. (https://doi.org/10.1210/jc.2016-2771)

Stuart CA, McCurry MP, Marino A, South MA, Howell ME, Layne AS, Ramsey MW \& Stone MH 2013 Slow-twitch fiber proportion in skeletal muscle correlates with insulin responsiveness. Journal of Clinical Endocrinology and Metabolism 98 2027-2036. (https://doi. org/10.1210/jc.2012-3876)

Usui T, Kajita K, Kajita T, Mori I, Hanamoto T, Ikeda T, Okada H, Taguchi K, Kitada Y, Morita H, et al. 2014 Elevated mitochondrial biogenesis in skeletal muscle is associated with testosterone-induced body weight loss in male mice. FEBS Letters 588 1935-1941. (https:// doi.org/10.1016/j.febslet.2014.03.051)

Wewege MA, Thom JM, Rye KA \& Parmenter BJ 2018 Aerobic, resistance or combined training: a systematic review and meta-analysis of exercise to reduce cardiovascular risk in adults with metabolic syndrome. Atherosclerosis 274 162-171. (https://doi.org/10.1016/j. atherosclerosis.2018.05.002)

White JP, Gao S, Puppa MJ, Sato S, Welle SL \& Carson JA 2013 Testosterone regulation of Akt/mTORC1/FoxO3a signaling in skeletal muscle. Molecular and Cellular Endocrinology 365 174-186. (https:// doi.org/10.1016/j.mce.2012.10.019)

Wu Y, Zhao W, Zhao J, Zhang Y, Qin W, Pan J, Bauman WA, Blitzer RD \& Cardozo C 2010 REDD1 is a major target of testosterone action in preventing dexamethasone-induced muscle loss. Endocrinology 151 1050-1059. (https://doi.org/10.1210/en.2009-0530)

Zhang J, Phillips DI, Wang C \& Byrne CD 2004 Human skeletal muscle PPARalpha expression correlates with fat metabolism gene expression but not BMI or insulin sensitivity. American Journal of Physiology: Endocrinology and Metabolism 286 E168-E175. (https://doi. org/10.1152/ajpendo.00232.2003)

Zurlo F, Larson K, Bogardus C \& Ravussin E 1990 Skeletal muscle metabolism is a major determinant of resting energy expenditure. Journal of Clinical Investigation 86 1423-1427. (https://doi. org/10.1172/JCI114857)

Received in final form 19 February 2020

Accepted 5 March 2020

Accepted Manuscript published online 5 March 2020 https://joe.bioscientifica.com https://doi.org/10.1530/JOE-19-0532 (c) 2020 Society for Endocrinology Published by Bioscientifica Ltd. Printed in Great Britain 